\title{
Uncertainty treatment in expert information systems for maintenance policy assessment
}

\author{
P. Baraldi ${ }^{1}$, M. Compare ${ }^{1}$, E. Zio ${ }^{2,1, *}$ \\ ${ }^{1}$ Politecnico di Milano, Italy \\ ${ }^{2}$ Chair on Systems Science and the Energetic Challenge, European Foundation for New Energy-Electricité \\ de France, Ecole Centrale Paris and Supelec, France
}

*Corresponding Author: enrico.zio@ecp.fr, enrico.zio@supelec.fr, enrico.zio@polimi.it,

Abstract: This paper proposes a framework based on the Dempster-Shafer Theory of Evidence (DSTE), Possibility Theory (PT) and Fuzzy Random Variables (FRVs) to represent and propagate the epistemic uncertainty in the parameters of the component degradation-failure models used to evaluate the performance of maintenance policies. An example of application is given with reference to a check valve of a turbo-pump lubricating system in a Nuclear Power Plant, which is affected by fatigue and overtakes condition-based maintenance interventions.

Keywords: Maintenance, Uncertainty, Fuzzy Random Variables (FRVs), Possibility Theory (PT), DempsterShafer Theory of Evidence (DSTE).

\section{Introduction}

Processing of uncertainty is crucial in industrial applications and consequently in decision making processes [1]. In practice, it is often convenient to distinguish uncertainty due to the inherent variability of the phenomena of interest from that due to lack of precise knowledge [2]. The former type is referred to as aleatory, irreducible, stochastic or random uncertainty and describes the inherent variation associated with the physical system or the environment, the latter is referred to as epistemic, subjective or reducible uncertainty, and relates to the lack of precise knowledge of quantities or processes of the system or the environment. Although probability theory is well suited to handle stochastic uncertainty due to variability, it has been argued that the probabilistic approach may have some limitations in the representation and treatment of epistemic uncertainty in situations of poor knowledge, since it tends to force assumptions which may not be justified by the available information [3]. For example, ignoring whether a value of a parameter is more or less probable than any other value within a given range does not justify assuming a uniform probability distribution, which is the less informative probability distribution according to both the Laplace principle of insufficient reason and the maximum entropy criterion [4].

In this work, we consider alternative approaches to probability theory for the representation of epistemic uncertainty, such as Dempster-Shafer Theory of Evidence (DSTE) and Possibility Theory (PT). These 
approaches have been considered due to their ability in handling the uncertainty associated to the imprecise knowledge on the values of parameters used by expert information systems and for which reliable data are lacking. In this respect, it is worthy noticing that some research effort has been devoted to capture the relationships between DSTE, PT and probability theory, and a vivid research debate is still ongoing about the capability of Probability Theory in representing the epistemic uncertainty

For example, in [5], a new framework is proposed, which extends Bayesian Theory to perform probabilistic inference with uncertain evidence. The extension is based on an idealized view of inference in which observations are used to rule out possible valuations of the variables in a modeling space. On the contrary, in [6] probability is conceptualized at the 'betting' level where decisions are made, which is different from the 'credal' level, where we find the epistemic uncertainty we are dealing with in this work. A pignistic transformation is required to pass from the credal level to the betting level. In [6], the authors also provide a comparison between the Bayesian framework and the Transferable Belief Model, which highlights that they may lead to different results.

The strength of DSTE and PT lies in their capability of representing the epistemic uncertainty in a way less committed than that offered by probability theory. PT has been embraced to tackle a number of interesting issues pertaining to different fields such as graph theory [7], database querying [8], diagnostics [9], data analysis [10] and classification [11], agricultural sciences [12], probabilistic risk assessment (e.g., [13], [14]), etc. to cite a few. Analogously, applications of DSTE can be found in diverse domains such as signal and image processing [15], business decision making [16], pattern recognition [17], clustering [18], etc.

In spite of the liveliness of the research in the field, it seems fair to say that the non-probabilistic treatment of uncertainty within soft computing methods has not yet been exhaustively investigated. After all, given the relative immaturity and small size of research community working on non-probabilistic approaches, it is hardly fair to expect that these are elaborated from soft methods to the same extent of that of probability theory [19]. In this respect, two main considerations can be done on the basis of the authors' best knowledge:

- There is no work of the literature which performs an comprehensive comparison of the main techniques to represent and propagate the epistemic uncertainty together with the aleatory uncertainty, from a practical, engineering point of view. For example, an interesting comparison of PT, DSTE and probability theory is provided in [20], where a simple case study is introduced as a workbench to highlight the differences among those approaches. However, even in that case the comparison is not complete, as neither (type 1 or 2) fuzzy theory nor Bayesian probability theory are considered. In conclusion, the issue of comparing the different frameworks is still open and future research effort will be spent by the authors in this direction. On the other side, while doing this, it is important to bear in mind that, quoting Smets [6]:

"Uncertainty is a polymorphous phenomenon. There is a different mathematical model for each of its varieties. No single model fits all cases. The real problems when quantifying uncertainty is to recognize its nature and to select the appropriate model. The Bayesian model is only one of them. The TBM also only one of them. Each has its own field of applicability. Neither is always better than the other"

For example, in [21] a different technique has been proposed to cope with the maintenance assessment issue in the case in which a team of experts is available to provide the ill-defined parameters, whereas the method proposed in this work assumes that there is just one expert to give them. 
- PT has never been applied in the context of maintenance modeling, which is the core of this paper.

Maintenance is a key factor for safety, production, asset management and competitiveness. Establishing an optimal maintenance policy requires the availability of logic, mathematical and computational models for:

i) the evaluation of performance indicators characterizing a generic maintenance policy. Possible performance indicators are the production profit, the system mean availability, the maintenance costs, etc.

ii) the identification of the optimal maintenance intervention policy from the point of view of the identified performance indicators, while fulfilling constraints such as those regarding safety and regulatory requirements. In practice, this multi-objective optimization problem has to be faced in a situation in which some constraints and/or the objective functions are affected by uncertainty. To effectively tackle this problem, a number of approaches have been already propounded in the literature considering different framework for uncertainty representation: probability distributions in [22]-[24], fuzzy sets in [25] and [26], and plausibility and belief functions in [27], [28].

The present work aims at contributing to the above step $i$ ) by developing a methodology for maintenance performance assessment that properly processes the involved uncertainties. More specifically, we consider a situation in which:

- a stochastic model of the life of the component of interest, in terms of degradation process, failure behavior and maintenance interventions is known without any uncertainty. This is, for example, the case for the degradation process 'fatigue' which has been successfully modeled by means of gamma processes [29], Weibull distributions [30], Paris-Erdogan law [31], etc.

- The model of the component's behavior depends on a number of ill-defined parameters. With reference to the example of fatigue degradation, the gamma process, Weibull distribution and Paris-Erdogan law depend on parameters whose values are usually not precisely known. Moreover, knowledge of other model parameters such as those describing the maintenance effectiveness (e.g., the improvement of the component degradation), duration and cost may also be imprecise. This framework of analysis where the aleatory and epistemic components of the uncertainty are separated into two hierarchical levels is often referred to as 'level 2' approach or setting [38].

- Information about the ill-defined parameters is available only from experts; in particular, it is assumed that there is a single expert, who provides for every uncertain parameter a set of intervals, which contain its true value with different degrees of possibility.

Although methods for a priori evaluating the performance of a maintenance policy while taking into account the aleatory uncertainty on the future behavior of the component of interest have been investigated in the literature (see [32]-[35] for surveys), only few works (e.g., [36]) tackle the maintenance policy performance assessment problem considering the epistemic uncertainty on the maintenance model parameters. In this work, the information elicited from the expert is described by means of possibility distributions and propagated through the model by resorting to a method that exploits the concept of FRVs [3], [37] and DSTE.

Notice that other techniques have been proposed in the literature to represent the imprecision in the expert qualitative statements. For example, type-2 fuzzy theory allows describing the uncertainty in the model parameters given by the expert by means of a fuzzy set, and also the uncertainty in the shape of its 
membership function, again by a fuzzy set [39]. This latter would introduce the 'third-level' uncertainty in our framework, which requires additional computational complexity to propagate the epistemic uncertainty described by the type- 2 fuzzy set together with the aleatory uncertainty. Furthermore, this solution leaves open the question: shall we consider the uncertainty in the membership defining the type-2 fuzzy set?

A different approach to the problem of assessing the maintenance performance while properly processing the involved uncertainties has been proposed by the authors in [21], [40], where the uncertainty in the model parameters is represented by resorting to the DSTE theory. In particular, the main differences between this latter approach and that presented in this work are outlined in [21], with respect to the information elicited, the readability of the output, the computational effort required and the possibility of passing from the PT-FRV-based method to the DSTE-based one.

The method proposed in this work is illustrated with reference to an exponential, non-repairable, binary component. A practical case study is shown with reference to the degradation model of a check valve of a turbo-pump lubricating system in a Nuclear Power Plant.

The remainder of the paper is organized as follows. Section 2 describes the method to represent and propagate the uncertainties. Section 3 illustrates a case study, which is for reference firstly investigated assuming that there is no epistemic uncertainty affecting the parameters of the stochastic model. The FRVbased method is then applied in Section 4 to this case study to treat epistemic uncertainty. Finally, a discussion on the pros and cons of the method, as emerged from its application to the case study, concludes the work. The main aspects of DSTE and PT are briefly recalled in Appendix 1 for completeness of the paper.

\section{Uncertainty setting}

Let us consider a model $\underline{Z}=g(\underline{Y})$, where $\underline{Z}=\left(Z_{1}, Z_{2}, \ldots, Z_{O}\right)$ is the vector containing the $O$ output variables of interest, and $g(\cdot)$ is a function that models how $\underline{Z}$ depends on the $k$ uncertain variables $Y_{j}, j=1, \cdots, k$, of vector $\underline{Y}$; the uncertainty on these variables is characterized by known probability distributions $F_{Y_{j}}\left(y_{j} ; \boldsymbol{\theta}_{j}\right)$, $j=1,2, \cdots, k$, where $\boldsymbol{\theta}_{j}=\left\{\theta_{j, 1}, \ldots, \theta_{j, M_{j}}\right\}$, are the vectors containing the hyper-parameters of the corresponding probability distributions. Also these parameters are uncertain and the information to characterize this uncertainty is drawn from an expert. This framework of analysis where the aleatory and epistemic components of the uncertainty are separated into two hierarchical levels is often referred to as 'level 2' approach or setting [39].

As mentioned, information is elicited from an expert for estimating the parameters $\boldsymbol{\theta}_{j}, j=1, \ldots, k$. The associated uncertainty is represented within the framework of PT, and propagated by means of the method based on the concept of FRVs. For the sake of clarity of the illustration, the treatment of uncertainty is described by ways of a simple case study concerning a non-repairable component whose state can only be either working or failed and whose Time To Failure $(T T F)$ is exponentially distributed with uncertain failure rate $\lambda$. The mission time is $T$ (taken equal to $10^{5} \mathrm{~h}$ in the numerical case study). Hence, in this reference example there is $k=1$ uncertain variable, i.e., $\underline{Y}=\left(Y_{1}\right)=(T T F)$, described by the Cumulative Distribution Function $(\mathrm{CDF}) F_{T T F}(t t f ; \lambda)=1-e^{-\lambda \cdot t f f}$, with $M_{1}=1$ uncertain parameter $\boldsymbol{\theta}_{1}=\{\lambda\}$. The output vector $\underline{Z}$ contains only one variable: the portion $D$ of the mission time in which the component is in a down state, i.e., unavailable. The function $g$ that links $T T F$ to $D$ is given by: 
$D=g(T T F)=\left\{\begin{array}{ccc}\frac{T-T T F}{T} & \text { if } & T T F \leq T \\ 0 & \text { otherwise } & \end{array}\right.$

Then, $D$ is also a random variable, because it is a function of the random variable TTF. The range of variability of $D$ is the interval [0,1], and its distribution, for a given value of the failure rate $\lambda$, is:

$$
F_{D}(d \mid \lambda)=P(D \leq d \mid \lambda)=P\left(\frac{T-T T F}{T} \leq d \mid \lambda\right)=P(T T F \geq T(1-d) \mid \lambda)=e^{-\lambda T(1-d)}
$$

where $d$ represents the generic value taken by the variable $D$.

Figure 1 shows the shape of this function for a value of the failure rate $\lambda=10^{-5} h^{-1}$. Notice that $F_{D}(0)$ (i.e., the probability that the component is always available during the mission time) is equal to $e^{-\lambda T}$, i.e., the probability that the component fails after $T$.

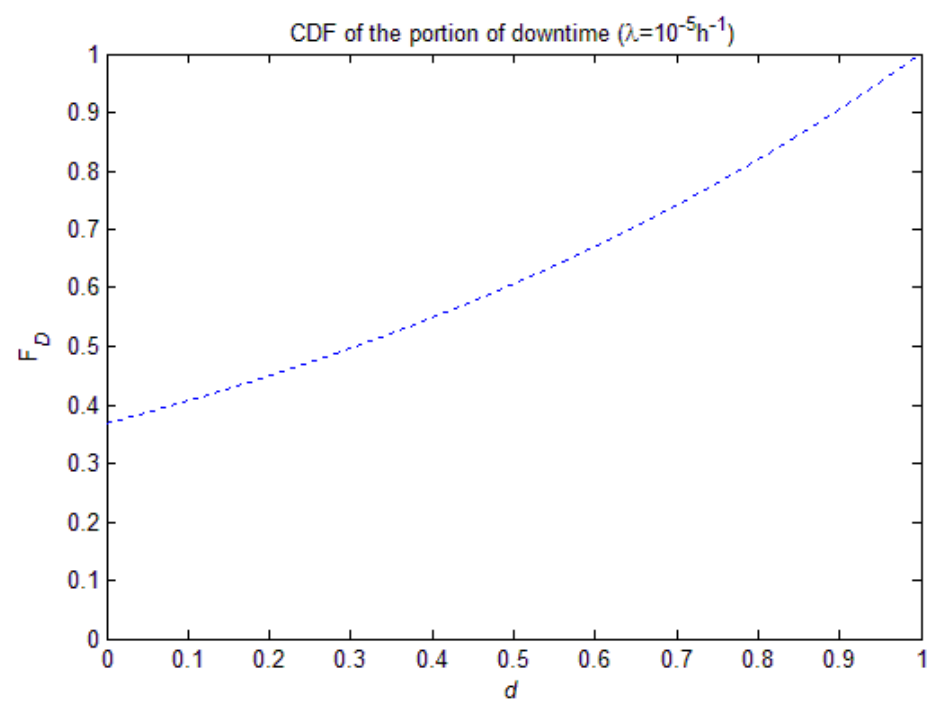

Figure 1: CDF of $D$, for $\lambda=10^{-5} \mathrm{~h}^{-1}$

\subsection{Information elicited from the expert}

Within the PT framework, for a generic uncertain parameter $\theta$, an expert is asked to provide a set of $n$ nested intervals $A_{i}, i=1, \ldots, n,\left(A_{1} \subseteq A_{2} \subseteq \ldots \subseteq A_{n}\right)$, which are believed to contain the true value of $\theta$ with a positive confidence level $q_{i}$; this latter can be conveniently interpreted as the smallest (subjective) probability that the true value of the parameter $\theta$ lies within $A_{i}$ (i.e., $\left.P\left(\vartheta \in A_{i}\right) \geq q_{i}\right)$. Alternatively, the interval $A_{i}$ can be seen as the smallest one whose probability of including the true value of $\theta$ is at least $q_{i}$ [41], for any $i=1, \ldots, n$. From the expert's point of view, $q_{i}$ is the portion of cases where $\theta \in A_{i}$ from his/her experience [41]. To sum up, the expert provides a weighted family $\left\{\left(A_{1}, q_{1}\right),\left(A_{2}, q_{2}\right), \ldots .,\left(A_{\mathrm{n}}, q_{n}\right)\right\}$ (for example, see Figure 2 (a)). Notice also that the value of the largest confidence level $q_{n}$ may be smaller than 1, i.e., $q_{n}=1-\varepsilon, \varepsilon>0$; this is equivalent to admitting that even the widest, safest interval contains some residual uncertainty $(\varepsilon)$, i.e., it is assumed that the expert is not absolutely sure about his judgment [41]. 
Finally, the inequalities $q_{1} \leq q_{2} \leq \ldots \leq q_{n}$ hold, due to the fact that $q_{i}$ of the interval $A_{i}$ is necessarily smaller than $q_{i+1}$ associated to $A_{i+1}$, if $A_{i} \subseteq A_{i+1}$, for any $i=1, \ldots, n-1$.
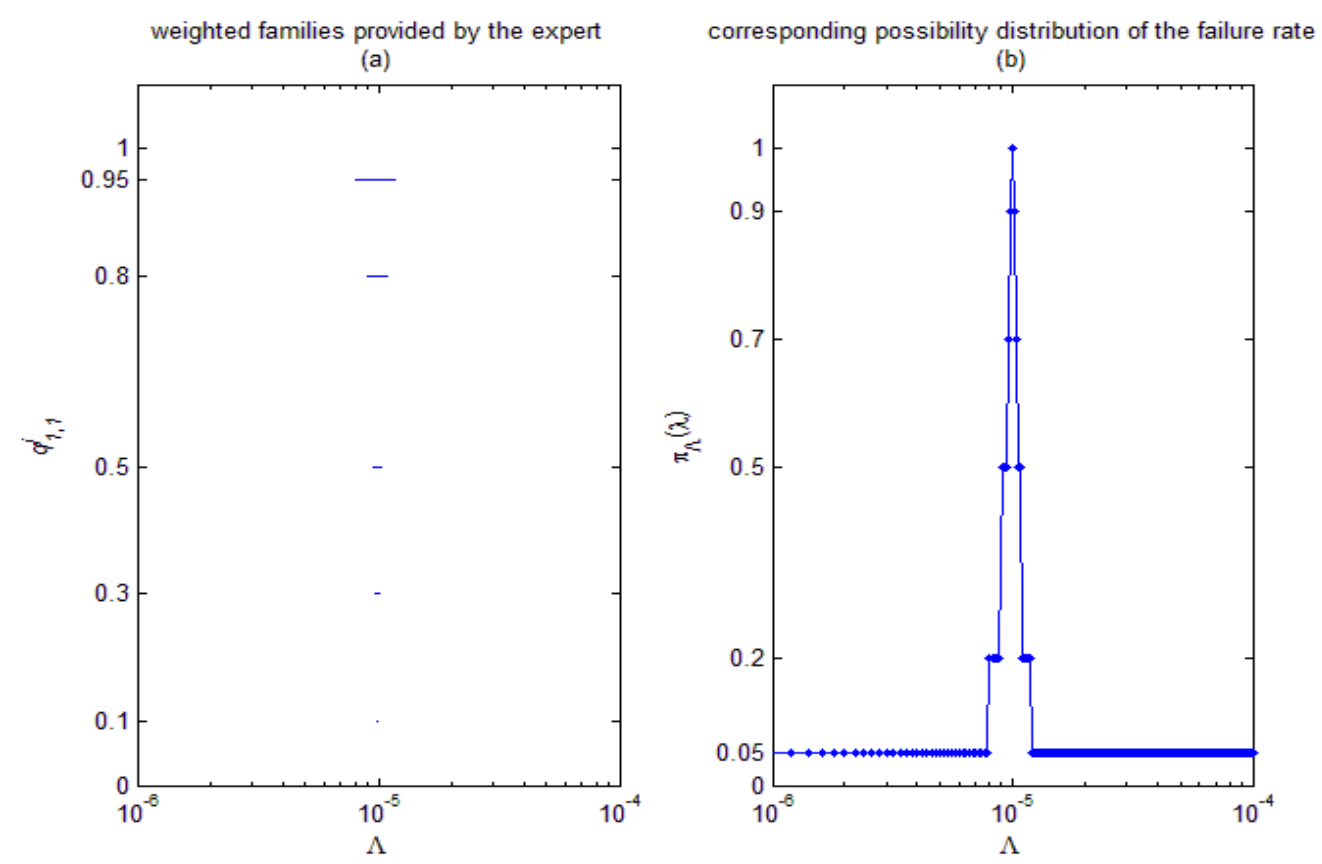

Figure 2: representation of the weighted families provided by the expert for the exponential, non-repairable, binary component (a) and the corresponding possibility distribution, built according to the procedure proposed in [43] (b)

With reference to the simple case study of the exponential, non-repairable, binary component, let us suppose that the expert characterizes his/her knowledge about the value of the failure rate $\lambda$ with the information summarized in Table 1.

\begin{tabular}{|c|c|c|c|c|c|c|c|c|c|c|}
\hline \multirow{3}{*}{ Parameters } & \multicolumn{10}{|c|}{ Degree of certainty } \\
\hline & \multicolumn{2}{|c|}{$q_{1}=0.1$} & \multicolumn{2}{|c|}{$q_{2}=0.3$} & \multicolumn{2}{|c|}{$q_{3}=0.5$} & \multicolumn{2}{|c|}{$q_{4}=0.7$} & \multicolumn{2}{|c|}{$q_{5}=0.95$} \\
\hline & $\min$ & $\max$ & $\min$ & $\max$ & $\min$ & $\max$ & $\min$ & $\max$ & $\min$ & $\max$ \\
\hline$\lambda\left[\mathrm{h}^{-1}\right]$ & $9.9 e-6$ & $1.01 \mathrm{e}-5$ & $9.7 e-6$ & $1.03 e-5$ & $9.5 e-6$ & $1.05 e-5$ & $9 e-6$ & $1.1 \mathrm{e}-5$ & $8 e-6$ & $1.2 \mathrm{e}-5$ \\
\hline
\end{tabular}

Table 1: information supposed to be elicited from the expert for the case study concerning the exponential, non-repairable, binary component

The Universe of Discourse (UoD), i.e., the interval of all the possible values of the failure rate is $[0, \infty[$, where the lower bound (0) corresponds to an infallible component, whereas an infinite failure rate corresponds to a component that fails at $t=0^{+}$. From Table 1, it appears that the expert provides the interval $A_{1}$ that is believed to normally, unsurprisingly contain the true failure rate value, with confidence level $q_{1}=0.1$, which represents the portion of cases where $\lambda \in A_{1}$ from the expert's experience point of view. The interval $A_{1}=\left[9.9 \mathrm{e}-6 \mathrm{~h}^{-1}, 1.01 \mathrm{e}-5 \mathrm{~h}^{-1}\right]$ is 'unsurprising' in the sense that any interval $A_{1}^{*}$ of the same length of $A_{1}$ would have been associated to an equal or smaller frequency of occurrence of the event $\lambda \in A_{1}^{*}$. Obviously, the expert cannot be less confident that the true value of the failure rate belongs to intervals that include $A_{1}$; thus, larger intervals are associated to larger confidence levels. In particular, $\mathrm{A}_{5}=\left[8 \mathrm{e}-6 \mathrm{~h}^{-1}, 1.2 \mathrm{e}-\right.$ $\left.5 \mathrm{~h}^{-1}\right]$ is the interval which leaves a $\varepsilon=0.05$ probability of not including the true value of $\lambda$. 
Figure 2(a) reports the set of intervals provided by the expert, and corresponding confidence levels (degrees of certainty). For visualization, both Figure 2(a) and Figure 2(b) report only the interval [1e- $\left.6 h^{-1}, 1 e-4 h^{-1}\right]$, instead of the entire UoD, and the abscissa axes are logarithmically scaled.

From Figure 2(b), it also emerges that the elicitation process should be checked against the overconfidence problem, which intervenes when judges provide intervals such that they were $\mathrm{X} \%$ sure that the correct answer lay between them, but the correct answer fell inside their intervals much less than X\% of the time. In this respect, the results of the study in [44] allow stating that the overconfidence problem is reduced or avoided when experts assign a reasonably accurate level of confidence to a given interval. On the contrary, when experts are asked to give an interval corresponding to a target assigned level of confidence the correctness of the judgment worsens. Thus, the findings in [44] seem to support the elicitation method proposed in this work.

With respect to the elicitation exercise of failure rates, a practical difficulty is given by the typically very low failure rate values (even lower than $10^{-6}$ which are difficult to directly assess resorting to expert knowledge. Alternatively, the experts can assess the mean time between failures, which is equal to the inverse of the failure rate and can be obtained from the ratio between the total time of observation and the number of failures occurred in such time span [45].

Notice that the elicitation exercise of the failure rate is not difficult from the expert point of view. In fact, it can be derived as the inverse of

More generally, in the uncertainty setting with $k$ variables, an expert is asked to provide for every $j=1, \ldots, k$ and $p=1, \ldots, M_{j}$, a set of $n_{j, p}$ nested intervals $A_{i}^{j, p}, i=1, \ldots, n_{j, p},\left(A_{1}^{j, p} \subseteq A_{2}^{j, p} \subseteq \ldots \subseteq A_{n_{j, p}^{j, p}}\right)$, which are believed to contain the true value of the $p$-th parameter of the $j$-th random variable, $\theta_{j, p}$, with a positive confidence level $q_{i}^{j, p}$.

\subsection{Uncertainty representation}

In this work, the uncertainty on the information elicited from the experts is represented by resorting to the possibility theory (see Appendix 1). With respect to an uncertain generic parameter $\theta$, the possibility theory defines, for a given set $A$, the possibility and necessity measures, $\Pi(A)$ and $N(A)$, which can be interpreted as the upper and lower limits of the probability that the true value of the parameter belongs to $A$. These two measures are related to the possibility distribution $\pi_{\theta}(\vartheta)$, which expresses the degree of possibility that the true value of the uncertain parameter be $\vartheta$, by:

$$
\begin{aligned}
& \Pi(A)=\sup _{\vartheta \in A}\left\{\pi_{\theta}(\vartheta)\right\} \\
& N(A)=1-\Pi(\operatorname{not}(A))=1-\sup _{\vartheta \nsubseteq A}\left\{\pi_{\theta}(\vartheta)\right\}
\end{aligned}
$$

In our case, a possibility distribution is directly built from the weighted families $\left\{\left(A_{1}, q_{1}\right),\left(A_{2}, q_{2}\right), \ldots,\left(A_{n}, q_{n}\right)\right\}$ provided by the expert, according to the procedure proposed in [41] and whose steps are here briefly recalled, for convenience. 
- First of all, it is postulated that the necessity measure, $N\left(A_{i}\right)$, i.e., the lower probability that the true value of $\theta$ is in the interval $A_{i}$, is equal to the confidence level $q_{i}$ defined by the expert. Thus, the inequality $P\left(\vartheta \in A_{i}\right) \geq N\left(A_{i}\right)=q_{i}$ holds, for any $i=1, \ldots, n$.

- Then, since there are infinite possibility distributions $\pi_{\theta}(\vartheta)$ that obey the constraint $q_{i}=N\left(A_{i}\right)$, it has been decided to choose the one which maximizes the degree of possibility $\pi_{\theta}(\vartheta)$ for all the values $\vartheta$. The solution is unique and is [4]:

$$
\pi_{\theta}(\vartheta)=\left\{\begin{array}{cc}
1 & \vartheta \in A_{1} \\
\min \left(1-q_{i}\right) & \text { otherwise } \\
i: \theta \notin A_{i} &
\end{array}\right\}
$$

In particular, it is possible to show that this is the least specific possibility distribution with respect to the available data, i.e., any other possibility distribution $\pi_{\theta}{ }^{\prime}$ obeying the constraints $q_{i}=\mathrm{N}\left(\mathrm{A}_{\mathrm{i}}\right)$ is such that $\pi_{\theta}{ }^{\prime} \leq \pi_{\theta}[4]$.

With reference to the case of the exponential, non-repairable, binary component, the possibility distribution $\pi_{\Lambda}$ of the failure rate $\lambda$ associated to the weighted family of Table 1 and built according to the procedure depicted above, is reported in Figure 2(b). To verify that this distribution obeys the constraints $q_{i}=N\left(A_{i}\right)$ for $i=1, \ldots, 5$, let us consider, for example, the first interval $A_{1}$; then, $N\left(A_{1}\right)=1-\Pi\left(n o t A_{1}\right)=1-\sup _{\vartheta \notin A_{1}}\left\{\pi_{\theta}(\vartheta)\right\}=1-0.9=0.1=q_{1}$. Notice also the residual uncertainty $\varepsilon=0.05$ associated to the points of the UoD external to $A_{5}$.

\subsection{Uncertainty propagation}

The uncertainty in the parameters of the model needs to be propagated to assess the uncertainty on the outputs. To this aim, we exploit the concept of FRVs within the methodology proposed in [3]. FRVs can be intuitively conceptualized as random variables whose values are not real numbers, but fuzzy numbers, since there is a vague perception of their true values, which are crisp but unobservable [37]. In other words, a FRV is a generalization of a random variable or a fuzzy variable [42].

The operative steps of the uncertainty propagation procedure are reported in the following with reference to the case of the exponential, non-repairable, binary component. Since this case is characterized by a single uncertain variable $(k=1)$, we will always omit in the notations the subscript 1 referred to the uncertain variable. As an example, the general procedure is given with reference to the sample vector $\left\{u_{j}^{\omega}\right\}$, but in our example we indicate it by $\left\{u^{\omega}\right\}$.

1) For each uncertain variable $Y_{j}, j=1,2, \cdots, k$, sample a vector $\left\{u_{j}^{\omega}\right\} \quad \omega=1,2, \cdots, N_{T}$, made of $N_{T}$ uniform random numbers in $[0,1[$; for example in our case, since $k=1$, we need a vector of random numbers $\left\{u^{\omega}\right\}, \omega=1,2, \cdots, N_{T}$. In particular, let us assume that the first sampled value $u^{1}=0.65$.

2) Select a value of $\alpha_{i}$ on [0,1] and take as intervals of possible values the cuts $\left[\underline{\boldsymbol{\theta}}_{j}, \overline{\boldsymbol{\theta}}_{j}\right]_{\alpha_{i}}=\left\{\left[\underline{\theta}_{j, 1}, \bar{\theta}_{j, 1}\right]_{\alpha_{i}}, \ldots,\left[\underline{\theta}_{j, M_{j}}, \bar{\theta}_{j, M_{j}}\right]_{\alpha_{i}}\right\}$ corresponding to the possibility distributions of the 
parameters $\boldsymbol{\theta}_{j}=\left\{\theta_{j, 1}, \ldots, \theta_{j, M_{j}}\right\}$, of the variables $Y_{j}, j=1,2, \cdots, k$; in our case, let us start from $\alpha_{i}=1$ : the interval of possible values for the parameter $\lambda$ is $\left[9.9 \mathrm{e}-6 \mathrm{~h}^{-1}, 1.01 \mathrm{e}-5 \mathrm{~h}^{-1}\right]$ (see Figure $2(\mathrm{~b})$ ).

3) Identify the set of random intervals $\left[\underline{y}_{j}^{\omega}, \bar{y}_{j}^{\omega}\right]_{\alpha_{i}}$, of the variables $Y_{j}, j=1, \ldots, k$, corresponding to the random vector $\left\{u_{1}^{\omega}, \ldots, u_{j}^{\omega}, \ldots, u_{k}^{\omega}\right\}$, using the $\alpha_{i}$-cut $\left[\underline{\boldsymbol{\theta}}_{j}, \overline{\boldsymbol{\theta}}_{j}\right]_{\alpha_{i}}=\left\{\left[\underline{\theta}_{j, 1}, \bar{\theta}_{j, 1}\right]_{\alpha_{i}}, \ldots,\left[\underline{\theta}_{j, M_{j}}, \bar{\theta}_{j, M_{j}}\right]_{\alpha_{i}}\right\}$, found at step 2). In particular, the $\omega$-th random interval of the $j$-th variable, $\left[\underline{y}_{j}^{\omega}, \bar{y}_{j}^{\omega}\right]_{\alpha_{i}}=\left[\inf _{\boldsymbol{\theta}_{j} \in\left[\underline{\theta}_{j}, \boldsymbol{\theta}_{j}\right]_{\alpha_{i}}} F_{Y_{j}}{ }^{-1}\left(u_{j}^{\omega} ; \boldsymbol{\theta}_{j}\right), \sup _{\boldsymbol{\theta}_{j} \in\left[\underline{\theta}_{j}, \overline{\boldsymbol{\theta}}_{j}\right]_{\alpha_{i}}} F_{Y_{j}}{ }^{-1}\left(u_{j}^{\omega} ; \boldsymbol{\theta}_{j}\right)\right]$, where $F_{Y_{j}}^{-1}\left(u_{j}^{\omega} ; \boldsymbol{\theta}_{j}\right)$ is the quasi-inverse function of the $\operatorname{CDF} F_{Y_{j}}\left(y_{j} ; \boldsymbol{\theta}_{j}\right)$ of the random variable $Y_{j}$, for any value of the vector $\boldsymbol{\theta}_{j}$ (i.e., if $U$ is a random variable uniformly distributed on $\left[0,1\left[\right.\right.$, then $F_{Y_{j}}{ }^{-1}\left(U ; \boldsymbol{\theta}_{j}\right)$ has $\left.\operatorname{CDF} F_{Y_{j}}\left(y_{j} ; \boldsymbol{\theta}_{j}\right)\right)$. This procedure can be regarded as an extension of the Monte Carlo (MC) sampling method, modified to take into account the fact that the parameters of the CDFs are fuzzy-uncertain in their UoDs: each sample from the uniform distribution is associated to an interval of values, instead of a single value (Figure 3), so that different CDFs are obtained from the sampling, and lower and an upper bounding CDFs can be identified.

In the reference case study, the interval associated to the sample $u^{1}=0.65$ and $\alpha_{i}=1$ is $[\underline{t t f}, \overline{t t f}]_{1}$ $=[1.63 \mathrm{e} 5 \mathrm{~h}, 1.66 \mathrm{e} 5 \mathrm{~h}]$ (Figure 3(a)). This is obtained by considering the two extremes of the interval of the uncertain parameter $\lambda$ equal to $[\underline{\theta}, \bar{\theta}]_{1}=\left[9.9 \mathrm{e}-6 \mathrm{~h}^{-1}, 1.01 \mathrm{e}-5 \mathrm{~h}^{-1}\right]$, which define the upper and lower exponential distributions, $1-e^{-\bar{\theta} t}$ and $1-e^{-\underline{\theta} t}$, respectively. Then, these functions are inverted to find the interval $[\underline{t t f}, \overline{t t f}]_{1}$, which is given by:

$$
[\underline{t t f}, \overline{t t f}]_{1}=\left[\frac{-\ln \left(1-u^{1}\right)}{\bar{\theta}}, \frac{-\ln \left(1-u^{1}\right)}{\underline{\theta}}\right] \text {. }
$$

Notice that in this particular case, the interval $[t t f, \overline{t t f}]_{1}$ is trivially obtained, since the inverse function of the exponential distribution is known. In general, it may be difficult to find out the analytical expression of the minimum and maximum values of the inverse function $F_{Y_{j}}{ }^{-1}\left(U ; \boldsymbol{\theta}_{j}\right)$, especially if it depends on a large number of parameters (e.g., $M_{j}>4,5$ ). In these cases, one has to devise efficient methods for identifying the minimum and maximum values of the random variable corresponding to the different combinations of the uncertain parameters $\left[\underline{\boldsymbol{\theta}}_{j}, \overline{\boldsymbol{\theta}}_{j}\right]_{\alpha_{i}}=\left\{\left[\underline{\theta}_{j, 1}, \bar{\theta}_{j, 1}\right]_{\alpha_{i}}, \ldots,\left[\underline{\theta}_{j, M_{j}}, \bar{\theta}_{j, M_{j}}\right]_{\alpha_{i}}\right\}$. 

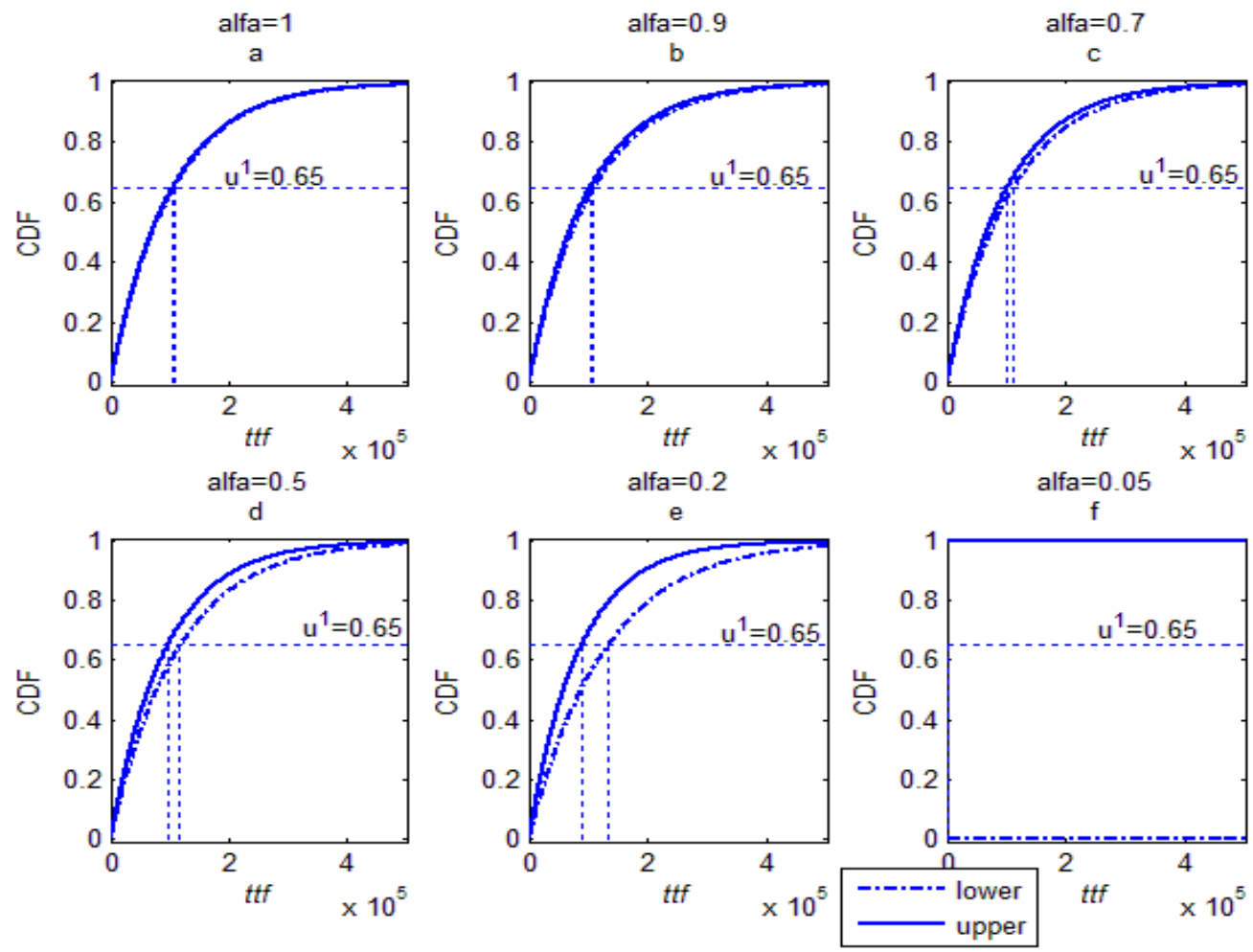

Figure 3: lower and upper CDFs corresponding to different values of $\alpha_{i}$, and the intervals associated by the quasiinverse functions to $u^{1}=0.65$

4) For every output variable $Z_{o}, o=1, . ., O$, calculate the smallest and largest values of $g$ (denoted by $\underline{g}_{\alpha_{i}}^{Z_{o}}(\omega)$ and $\bar{g}_{\alpha_{i}}^{Z_{o}}(\omega)$, respectively), within the intervals $\left[\underline{y}_{j}^{\omega}, y_{j}^{\omega}\right]_{\alpha_{i}}, j=1,2, \cdots, k$, of the variables:

$$
\begin{aligned}
& \underline{g}_{\alpha_{i}}^{Z_{o}}(\omega)=\inf _{\left.j, y_{j} \in \underline{\underline{y}}_{j}^{i}, y_{j}^{i}\right]_{\alpha_{i}}} g\left(y_{1}, \ldots, y_{j}, \ldots, y_{k}\right) \\
& \bar{g}_{\alpha_{i}}^{Z_{o}}(\omega)=\sup _{j, y_{j} \in\left[\underline{y}_{j}^{i}, y_{j}^{i}\right]_{\alpha_{i}}} g\left(y_{1}, \ldots, y_{j}, \ldots, y_{k}\right)
\end{aligned}
$$

for $o=1, \ldots, O$, and consider the interval:

$$
\Gamma_{\alpha_{i}}^{z_{o}}(\omega)=\left[\underline{g}_{\alpha_{i}}^{z_{o}}(\omega), \bar{g}_{\alpha_{i}}^{Z_{o}}(\omega)\right]
$$

In the case of the exponential, non-repairable, binary component, the minimum and maximum values of the $T T F$ found in the previous step (i.e., $1.63 \mathrm{e} 5 \mathrm{~h}$ and $1.66 \mathrm{e} 5 \mathrm{~h}$, respectively) are both larger than the mission time $T=10^{5} \mathrm{~h}$, and thus the corresponding values of $D$ are zero (Equation 1).

5) Return to step 2) and repeat steps 3) and 4) for another $\alpha$-cut. For the exponential, non-repairable, binary component, the intervals $\left[t t f_{1}^{1}, \overline{t t f}_{1}^{1}\right]_{\alpha_{i}}$ corresponding to different values of $\alpha_{i}$ are reported in

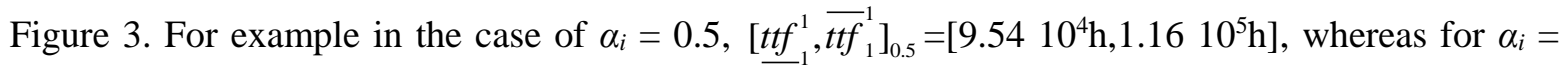
0.05 the interval $\left[\underline{t t f}_{1}^{1}, \overline{t t f}_{1}^{1}\right]_{0.05}=[0, \infty[$.

6) The FRV corresponding to the $\omega$-th realization is computed as:

$$
\pi_{Z_{o}(\omega)}\left(z_{o}\right)=\sup \left[\alpha_{i} \in[0,1] \mid z_{o} \in \Gamma_{\alpha_{i}}^{Z_{o}}(\omega)\right]
$$


The FRV that describes the portion of the component downtime associated to the first sample is shown in Figure 4: since $\pi_{D(1)}(0)=1$ it is fully plausible that the component is available for the overall mission time, whereas, since $\pi_{D(1)}(1)=0.05$ it is not impossible that the component is unavailable for the entire mission time. Furthermore, according to the probabilistic interpretation of the possibility distribution, it is possible to observe that the probability that the component is fully available during its mission time is between 0.5 and 1 , and the probability that the portion of downtimes is larger than 0.07 is between 0 and 0.2. Notice that the FRV of Figure 4 is consistent with the intervals represented in Figure 3. In fact, only the intervals corresponding to $\alpha_{i} \leq 0.5$ contain the value $T=10^{5} \mathrm{~h}$ : this means that only for these values of $\alpha_{i}$ the component may experience a failure before $T$, which entails its unavailability for the remaining part of the mission time.

7) Repeat steps 1)-6) for a new realization of the random variables, until $\omega=N_{T}$.

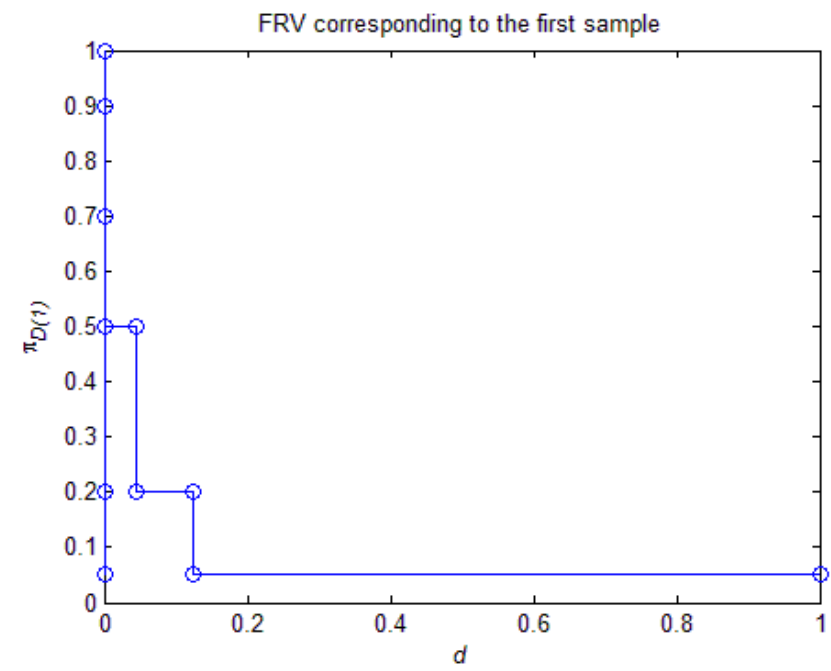

Figure 4: Fuzzy Random Variable corresponding to the sample $u^{1}=0.65$

8) Compute the Plausibility and Belief distributions for $Z_{o}, o=1, \ldots, O$ by:

$$
\begin{aligned}
& \left.\left.\operatorname{Pl}\left(Z_{o} \in\right]-\infty, z_{o}\right]\right)=\sum_{\omega=1}^{N_{T}} \frac{1}{N_{T}} \cdot \sup _{\left.\left.z_{o} \in\right]-\infty, z_{o}\right]} \pi_{Z_{o}(\omega)}\left(z_{o}\right), \\
& \left.\left.\operatorname{Bel}\left(Z_{o} \in\right]-\infty, z_{o}\right]\right)=\sum_{\omega=1}^{N_{T}} \frac{1}{N_{T}} \cdot \inf _{\left.\left.z_{o} \notin\right\}-\infty, z_{o}\right]}\left(1-\pi_{Z_{o}(\omega)}\left(z_{o}\right)\right)
\end{aligned}
$$

where $1 / N_{T}$ is the probability assigned to the $\omega$-th FRV, for any $\omega$. In particular, Equation 10 is derived from the interpretation of the FRVs under the setting of random sets [43].

The Plausibility and Belief distributions of $D$ (i.e., the upper and lower bounds, respectively, of the probability distributions of the portion of the mission time $T$ in which the exponential, non-repairable, binary component is in a fault state) are reported in Figure 5; for comparison, the CDF (see Equation. 2) is also provided, which lies between the Plausibility and Belief distributions.

A comment seems in order about the requirement that the uncertainties on all the input parameters must be described by the same expert, which is mandatory for applying this procedure. This constraint comes from the application of the extension principle in Equation 9, which introduces a strong dependence between the information sources supplying the input possibility distributions. Indeed, the same confidence level for all the input variables is chosen to build the $\alpha$-cuts of the output variables; this suggests that if the expert source informing on one parameter is rather precise or gives the same mean values to the confidence levels, then the 
one informing on another parameter must also be precise, i.e., to ensure this, it must be the same source. Further research effort should be spent in order to verify whether the procedure here illustrated can be interpreted as a conservative counterpart to the calculus of probabilistic variables under stochastic independence, due to the dependence between the choice of confidence levels.

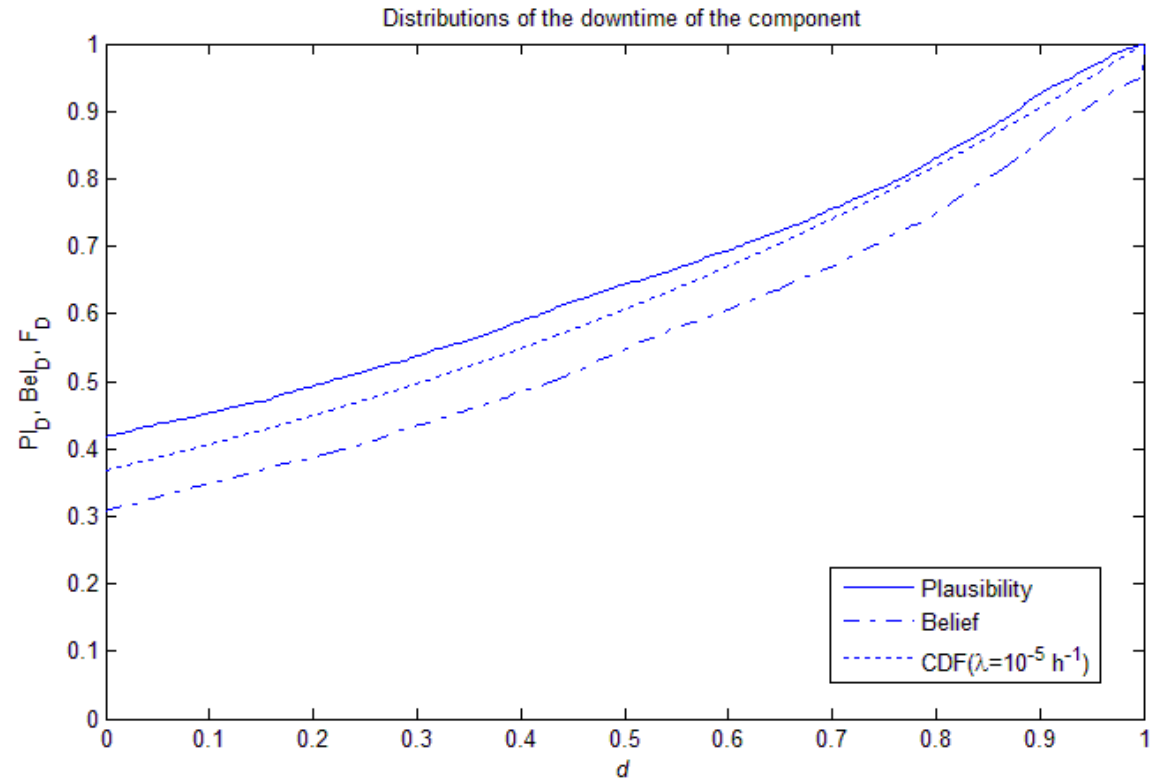

Figure 5: Plausibility, Belief and Cumulative distributions of the portion of mission time in which the component is in a fault state

\section{Case Study}

The present case study is taken from [46] and regards the degradation and maintenance of a check valve of a turbo-pump lubricating system in a Nuclear Power Plant. The degradation modelling is based on information collected from dependability analyses (e.g., FMECA) or directly from experts. This leads to the identification of one principal degradation mechanism, i.e., fatigue, and only one failure mode, i.e., rupture. A Condition-Based Maintenance (CBM) policy is applied to this component on a time horizon $T=10^{4} \mathrm{~h}$. The performance of the maintenance policy is assessed in terms of cost and component unavailability.

Notice that the case study proposed in this work is derived from a real industrial issue addressed by the authors. However, the values of the parameters of the degradation - failure model considered in this Section have been arbitrarily set. In fact, the focus of the paper is on the method proposed to cope with the issue of assessing a maintenance policy in the presence of uncertainty in the model parameters, rather than on the findings of the case study.

\subsection{Degradation mechanism modeling}

The fatigue phenomenon affecting the check valve is here modeled as a discrete-state, continuous-time stochastic process that evolves among the following three degradation levels (Figure 6):

1. 'Good': a component in this state is new or almost new (no crack is detectable by maintenance operators).

2. 'Medium': if the component is in this degradation level, then it is convenient to replace it.

3. 'Bad': a component in this degradation state is very likely to experience a failure in few working hours. 
The choice of describing the degradation process by means of a small number of levels, or degradation 'macro-states', is driven by industrial practice: experts usually adopt a discrete and qualitative classification of the degradation states based on qualitative interpretations of symptoms.

The probability density functions (pdfs) of the transition times are Weibull distributions, with scale parameters $\eta_{i j}$ and shape parameters $\beta_{i j}$ for the transitions from state $i$ to state $j(i, j \in\{1,2,3\}$, and $i<j)$. The Weibull distribution is commonly applied in fracture mechanics (e.g., [30]), especially under the weakestlink assumption [47].

A further state, 'Failed', can be reached from every degradation state upon the occurrence of a shock event. The exponential distribution with constant failure rate $\lambda_{j}$ describes the failure behaviour of the component while it is in state $j$, for every $j=1,2,3$. The choice of assigning a constant failure rate to every degradation state is driven by industrial practice: experts are familiar with this setting and comfortable with providing information about the failure rates values.

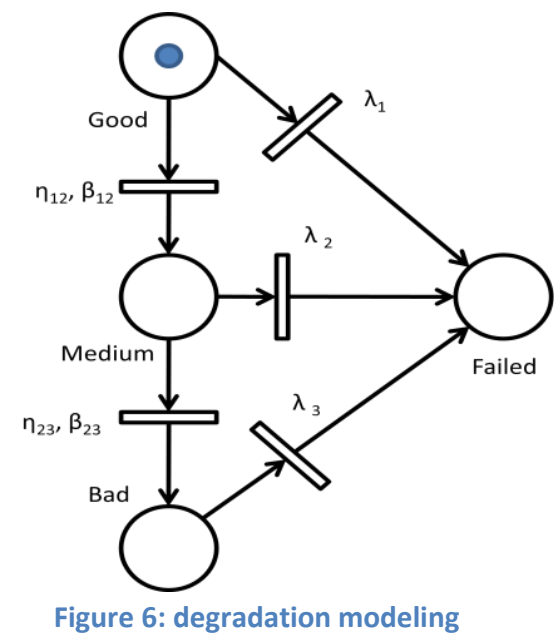

\subsection{Maintenance policy}

The CBM policy applied to the system is composed by the following tasks:

- Inspections: these actions, which are the only scheduled actions, are aimed at detecting the degradation state of the component, and are considered to last $5 \mathrm{~h}$ for a cost of $50 €$. For the sake of simplicity, the component is considered as new after the inspection.

- CBM actions: Preventive Maintenance (PM) actions which are dependent on the result of an inspection action. More precisely, if the component is found to be in state 'Good', no action is performed, whereas if the degradation state is 'Medium' or 'Bad', then the component is replaced and, consequently, the degradation state is taken back to 'Good'. Both these replacement actions are supposed to take $25 \mathrm{~h}$ and cost $500 €$, each.

- Corrective Maintenance (CM) actions. The corrective action, performed after a component failure, is assumed to be the replacement of the component. Due to the fact that this event is unscheduled, this action brings an additional duration of $85 \mathrm{~h}$ and an additional cost of $3500 €$, with respect to the replacement after an inspection, leading to a total duration of $100 \mathrm{~h}$ and to a total cost of $4000 €$. In particular, the additional time may be caused by the supplementary time needed for performing the procedure of replacement after failure or to the time elapsed between the occurrence of the failure and the start of the replacement actions. 
The Inspection Interval (II), which is the time span between two successive planned inspections, is the only decision variable considered in this case study; optimization is then directed to the search for the value of the II that minimizes the costs and maximizes the availability of the component.

Notice that to keep the case study presented in this work consistent with the practical industrial problem tackled by the authors, the values of duration and cost of the different maintenance actions are given proportional to the actual ones.

\subsection{Analysis of the case study}

The case study is firstly investigated in the unrealistic situation in which the values of the model parameters $\theta_{j, p}, j=1, \ldots, k$ and $p=1, \ldots, M_{j}$ are assumed to be exactly known (i.e., there is no epistemic uncertainty). Table 2 reports the values of these parameters, which have been taken from [46].

$\mid$\begin{tabular}{|c|c|}
\hline Parameters & Nominal Values \\
\hline$\theta_{1,1}=\eta_{12}$, & $1861 \mathrm{~h}$ \\
\hline$\theta_{1,2}=\beta_{12}$ & 8 \\
\hline$\theta_{2,1}=\eta_{23}$ & $743 \mathrm{~h}$ \\
\hline$\theta_{2,2}=\beta_{23}$ & 8 \\
\hline$\theta_{3,1}=\lambda_{1}$ & $10^{-6} \mathrm{~h}^{-1}$ \\
\hline$\theta_{4,1}=\lambda_{2}$ & $10^{-4} \mathrm{~h}^{-1}$ \\
\hline$\theta_{5,1}=\lambda_{3}$ & $10^{-2} \mathrm{~h}^{-1}$ \\
\hline
\end{tabular}
Table 2: Parameters of the probability distributions

Figure 7 shows the CDF of the portion of the mission time in which the component is unavailable. Two main steps can be observed, which can be explained by imaging to have a population of identical components. According to the MC simulation, almost $60 \%$ of the population experience one out of the following two behaviors.

- The component never fails during the mission time, and thus is inspected 4 times (at $t=2000 \mathrm{~h}$, $4000 \mathrm{~h}, 6000 \mathrm{~h}$ and $8000 \mathrm{~h}$ ); in 3 out of these 4 inspections the component is found in degradation states Medium or Bad (75h of downtime) and in the remaining one in degradation state Good (5h of downtime). Thus, the total downtime is $80 \mathrm{~h}$, which constitutes the $0.8 \%$ of $T$. Components experiencing this life explain the CDF step in correspondence of $d=0.008$.

- The component never fails during the mission time, and when inspected is always found in degradation states Medium or Bad (100h of downtime). This behavior explains the CDF step in correspondence of $d=0.01$. 


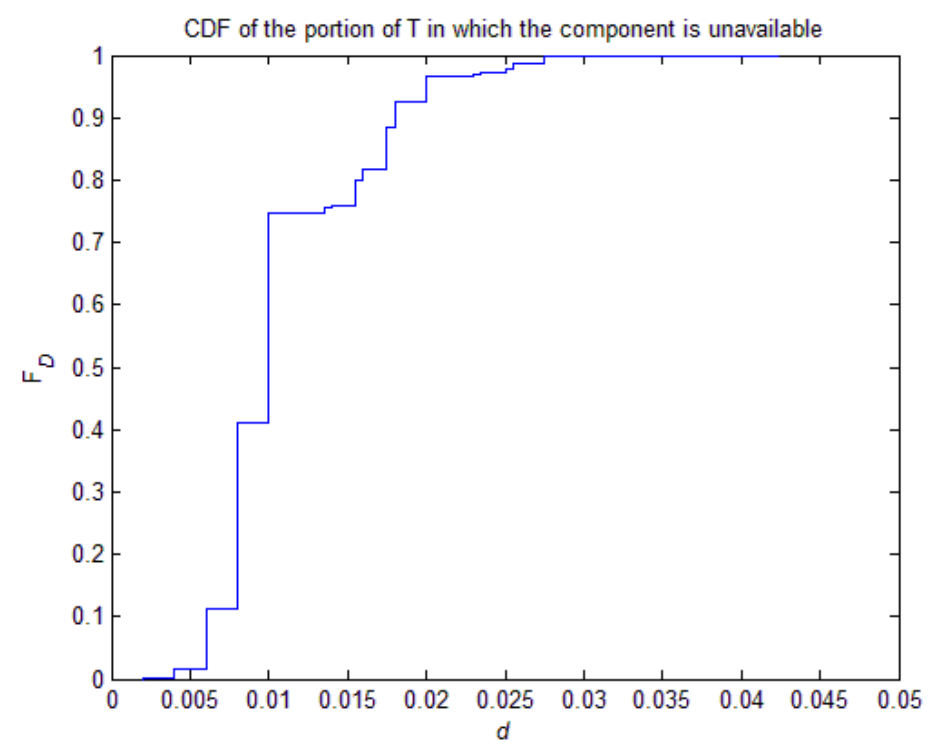

Figure 7: CDF of the portion of the time horizon in which the component is in a down state

Notice that it is possible to lump together the information provided by the cumulative distribution of the portion of downtimes into the mean value of the distribution, i.e., the average unavailability over the mission time, which provides an useful and easy to be interpreted indicator of the component expected state in the mission time. The estimated average unavailability is 0.011 , and the related $68.3 \%$ confidence intervals is [0.011-9.8e-8, 0.011+9.8e-8].

\subsection{Maintenance optimization}

Figure 8 shows the estimated average unavailability of the component over the mission time (i.e., the mean value of the component downtime over the entire mission time), with the related $68.3 \%$ confidence interval, for different values of the II. The narrowness of the confidence intervals is due to the large number $\left(5^{*} 10^{4}\right)$ of MC simulations performed in this case study; roughly speaking, the larger this number the smaller the (confidence) interval that with a given probability (confidence level) contains the true value of the estimated variable. Thus, in the present case study the actual value of the average unavailability over the mission time is affected by a small amount of error, which can be reduced by increasing the number of simulations. 


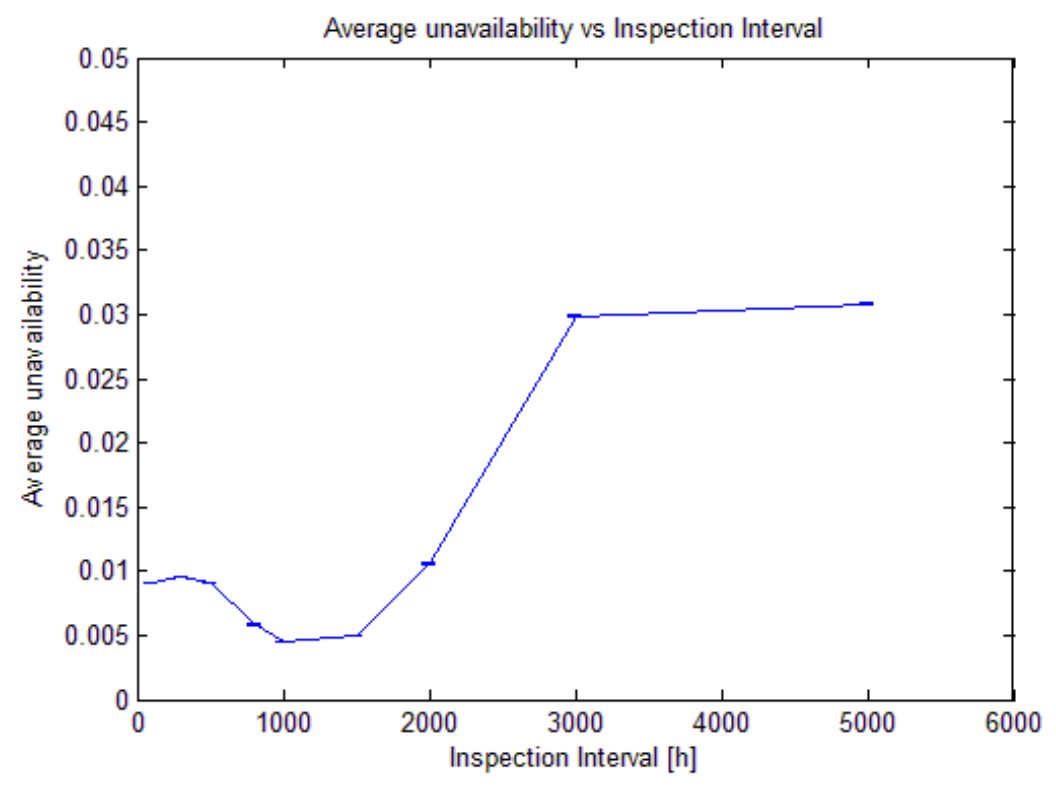

Figure 8: average unavailability corresponding to different Inspection Intervals

Initially, there is a decreasing behavior that reaches a minimum in correspondence of $I I=1000 \mathrm{~h} / 1500 \mathrm{~h}$; after this point, the unavailability starts rapidly increasing. This is the result of two conflicting trends: on one side, the more frequent are the inspections the larger is the probability to find the component in degradation states Medium and Bad: this prevents the component to fail and thus saves the corresponding large time to repair. On the other side, frequent replacements are ineffective, since the component life is not completely exploited in this case. The minimum at $I I=1500 \mathrm{~h}$ represents the optimal balance between these two tendencies.

Figure 9 shows that the maintenance costs associated to different choices of the $I I$ have a shape similar to that of the mean unavailability. Thus, one may conclude that under the considered maintenance policy, the best $I I$ is between $1000 \mathrm{~h}$ and $1500 \mathrm{~h}$ with respect to both availability and cost objectives. On the other hand, both the mean unavailability and the maintenance cost remain small, with little variations, when the value of the II ranges in the interval [1000h, 2000h]. This relative flatness of both performance indicators (unavailability and cost) allows a certain freedom to choose the $I I$ within such range: other criteria (e.g., opportunistic maintenance) not included in this analysis can be taken into account if the related advantages recover the small losses due to the increase of unavailability and cost, that would be incurred when moving away from the optimal value of $1500 \mathrm{~h}$. 


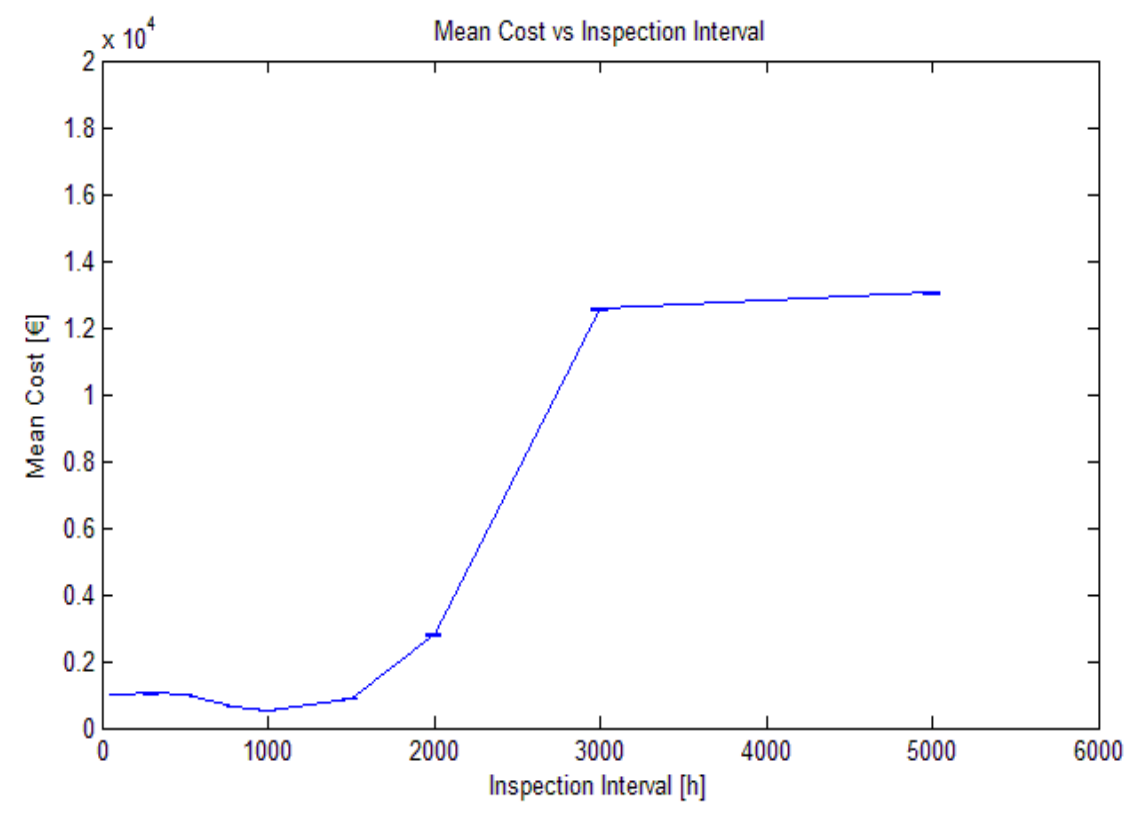

Figure 9: mean costs corresponding to different Inspection Intervals

\section{Representation and propagation of the uncertainties in the considered case study}

The aim of this Section is to apply the method illustrated in Section 2 to the case study described above, when the parameters of the distributions that model the transitions of the component among the four states of Figure 6 are ill-defined and there is only one expert who estimates their values. To sum up, the uncertainty situation is the following:

- $\quad$ there are $k=5$ uncertain variables, which define the 5 transition times reported in Table 3.

- The distributions associated to the variables are known, and depend on the set of the uncertain parameters $\theta_{j}, j=1 \ldots, 5$ reported in Table 3 . In turn, there are $N u=7$ uncertain parameters, which are the shape and scale parameters of the two Weibull distributions and the failure rates pertaining to the three degradation levels (see Table 3).

\begin{tabular}{|l|l|l|}
\hline $\begin{array}{l}\text { Uncertain } \\
\text { Variables }\end{array}$ & $\begin{array}{l}\text { Uncertain } \\
\text { Parameters }\end{array}$ & Description \\
\hline$Y_{1}$ & $\boldsymbol{\theta}_{1}=\left\{\theta_{1,1}, \theta_{1,2}\right\}$ & Transition time from degradation level 'Good' to 'Medium' \\
\hline$Y_{2}$ & $\boldsymbol{\theta}_{2}=\left\{\theta_{2,1}, \theta_{2,2}\right\}$ & Transition time from degradation level 'Medium' to 'Bad' \\
\hline$Y_{3}$ & $\boldsymbol{\theta}_{3}=\left\{\theta_{3,1}\right\}$ & Transition time from degradation level 'Good' to 'Failed' \\
\hline$Y_{4}$ & $\boldsymbol{\theta}_{4}=\left\{\theta_{4,1}\right\}$ & Transition time from degradation level 'Medium' to 'Failed' \\
\hline$Y_{5}$ & $\boldsymbol{\theta}_{5}=\left\{\theta_{5,1}\right\}$ & Transition time from degradation level 'Bad' to 'Failed' \\
\hline \multicolumn{2}{|c|}{ Table 3: tailoring of the general model to the considered case study } \\
\hline
\end{tabular}

Notice that the simulation of a single MC history (steps 1-5 of the procedure in Section 2.3) requires that the model $g$ encodes a number of random variables $k>>5$, since the history corresponding to a given sample of 
these 5 uncertain times in general do not cover the entire time horizon $T$. For example (Figure 10 (a)), if the first transition is from state 1 to state 'Failed' and occurs at $t=2000 \mathrm{~h}$, then the interval time between $t=2000 \mathrm{~h}+100 \mathrm{~h}$ (i.e., the time instant at the end of the repair action that starts after the failure) and $T$ remains not investigated. This problem can be overcome by thinking of $g$ as a function that depends on a number $K$ of 5 -ple (the 5 probabilistic variables), and not just on 5 variables. Obviously, the number $K$ that allows to cover the entire mission time is also a random variable, since it depends on the sampled times, which produce histories of different lengths. However, this is not a problem in practice: the number $K$ can be chosen such that it is reasonably sure that the sampled times simulate histories of duration larger than the time horizon $T$. Then, the analysis focuses only on the interval $[0, T]$ (Figure 10(b)). Finally, the output vector $\underline{Z}$ is made up

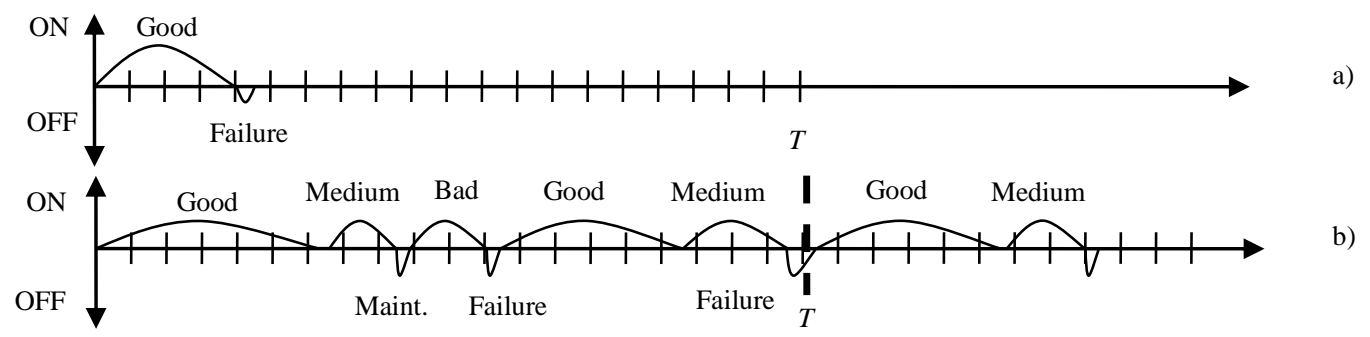

Figure 10:Two examples of simulated histories: the number of random variables does not suffice to cover the entire time horizon $T$ (a); number $K$ allows to simulate histories longer than $T$ (b)

of the portion of $T$ in which the component is unavailable, and the cost associated to the maintenance policy to be assessed; thus $O=2$.

\subsection{Single expert information}

In all generality, the difficulty in estimating the uncertain parameters of the model may heavily vary from one case to another; the weighted families $\left\{\left(A_{1}^{j, p}, q_{1}^{j, p}\right),\left(A_{2}^{j, p}, q_{2}^{j, p}\right), \ldots .,\left(A_{n_{j, p}}^{j, p}, q_{n_{j, p}}^{j, p}\right)\right\}$ provided by the expert to represent his/her knowledge about the parameters are expected to reflect this difference.

In regard to the considered case study, the weighted families elicited from the single expert are reported in Table 4. It is assumed that he/she is able to infer the information on the transition times between the different states, from the observations gathered during past component inspections. For example, let us suppose that a component is monitored every $100 \mathrm{~h}$, and that it was found in degradation state Medium a $t=1800 \mathrm{~h}$; if the component is found in degradation state Bad upon the next observation at $t=1900 \mathrm{~h}$, then the expert acquires the information that the transition from degradation state Medium to Bad occurred in the interval ]1800h, 1900h[. This kind of information can be used to define the scale and shape parameters of the Weibull distributions representing these transitions. In this respect, remind that the scale factor of a Weibull distribution is by definition the time before which almost 65\% (i.e., 63,2\%) of the components of an homogeneous population have experienced a transition, whereas the shape factor, roughly speaking, determines the uncertainty around the scale value. Notice, however, that the amount of uncertainty affecting the estimations of the scale and shape parameters is expected to be very different: the expert has a more refined knowledge on the scale parameter as it can be seen as the 65th percentile, than on the shape parameter which is only related to the slopes of the Weibull probability plots; these are expected to be difficult to estimate from the observations of the components' degradation states during the inspections. Anyway, it is the authors' experience that in some cases (e.g., the degradation behaviour of some components of the turbines used in the Oil\&Gas industry), the experts of the maintenance engineering department have a relatively precise knowledge about the values of the shape factors. 


\begin{tabular}{|c|c|c|c|c|c|c|c|c|c|}
\hline \multicolumn{2}{|c|}{} & \multicolumn{2}{|c|}{$q_{1}^{j, p}=0.1$} & \multicolumn{2}{c|}{$q_{2}^{j, p}=0.5$} & \multicolumn{2}{c|}{$q_{3}^{j, p}=0.95$} & \multicolumn{2}{c|}{ UoD } \\
\cline { 2 - 10 } & $\min$ & $\max$ & $\min$ & $\max$ & $\min$ & $\max$ & \multicolumn{2}{c|}{ Min } & $\max$ \\
\hline$\theta_{1,1}$ & $\eta_{12}$ & 1843 & 1880 & 1815 & 1908 & 1720 & 2001 & 1700 & 2020 \\
\hline$\theta_{1,2}$ & $\beta_{12}$ & & & & & 7.5 & 8.5 & 7 & 9 \\
\hline$\theta_{2,1}$ & $\eta_{23}$ & 735 & 750 & 725 & 762 & 687 & 800 & 650 & 850 \\
\hline$\theta_{2,2}$ & $\beta_{23}$ & & & & & 7.5 & 8.5 & 7 & 9 \\
\hline$\theta_{3,1}$ & $\lambda_{1}$ & & & & & $9 \mathrm{e}-7$ & $1.1 \mathrm{e}-6$ & $1 \mathrm{e}-7$ & $5 \mathrm{e}-6$ \\
\hline$\theta_{4,1}$ & $\lambda_{2}$ & & & & & $9 \mathrm{e}-5$ & $1.1 \mathrm{e}-4$ & $1 \mathrm{e}-5$ & $5 \mathrm{e}-4$ \\
\hline$\theta_{5,1}$ & $\lambda_{3}$ & & & & & $0.9 \mathrm{e}-2$ & $1.1 \mathrm{e}-2$ & $0.85 \mathrm{e}-2$ & $1.15 \mathrm{e}-2$ \\
\hline
\end{tabular}

Table 4: Confidence levels and associated intervals

With regards to the scale parameters (rows 4 and 6 of Table 4), it has been assumed that the expert provides three nested intervals corresponding to the confidence levels $q_{1}^{j, p}=0.1, q_{2}^{j, p}=0.5$ and $q_{3}^{j, p}=0.95, j=\{1,2\}$ and $p=1$, and the UoDs within which these parameters range.

With regards to the shape parameters (rows 5 and 7 of Table 4), given the difficulty in their estimation, it has been assumed that the expert provides just the UoDs and the intervals corresponding to the confidence level $q_{3}^{j, p}=0.95$. In particular, the UoDs, which contain the true values of the parameters with probability 1 , are very large: the expert tends to reduce the sets of values that surely do not contain the true values of the scale parameters.

Finally, with reference to the failure rates, also the estimation of the Mean Time To Failure (MTTF, i.e., the inverse of the failure rate) of the components in a given degradation state may not be easy; in fact, failure from the first degradation state is usually a rare event, whose frequency is difficult to estimate even in a qualitative way, whereas the lack of precise knowledge of the time instants in which the components transit towards the other degradation states affects the evaluation of the mean times to failure associated to these states; that is, if the time instant since one has to start to count is unknown, then the resulting measure of the time to failure is biased, especially if the component is rarely inspected. Thus, a more vague description of the uncertainty is provided by the expert for these parameters of the model $g$. Namely, he/she gives just the intervals corresponding to the 0.95 confidence level and the $\mathrm{UoD}$, as for the scale parameters of the Weibull distributions. The extreme points of these intervals are reported in rows $8-10$, columns 7-10 of Table 4 .

On the other side, the larger the number of the uncertain parameters, the larger the space in which the maxima and minima of the function $g$ in Equations 6 and 7 have to be searched for, and the larger the required computational time. In this regard, a sensitivity analysis can be preventively performed in order to identify which are the input parameters whose variations lead to smaller changes in the output value; this allows to neglect the uncertainty affecting these parameters while losing a small amount of information and considerably reducing the computational times.

In the present case study, the sensitivity analysis is performed by a local approach [51], i.e., the uncertain parameters of the model are varied one by one within their UoD, while the other parameters take their nominal values. The results of the analysis are reported in Table 5: the portion of $T$ in which the component is unavailable is estimated in correspondence of the extreme values of the UoD of every uncertain parameter. For example, the estimation of $D$ is 0.0142 in correspondence of the lower bound of the UoD of the scale parameter of the first Weibull distribution $(1700 \mathrm{~h})$, whereas it is 0.0082 in correspondence of the upper bound (2020h). In particular, these values are reported with the related $68.3 \%$ confidence interval. The last 
column of the Table reports $\Delta$, i.e., the differences between the average unavailability corresponding to the two limiting situations. These quantities give an estimation of the amount of output uncertainty (i.e., the unavailability uncertainty) due to the variation of the model parameters. In practice, high values of $\Delta$ indicate the importance of properly considering the uncertainty in the parameters, whereas low values correspond to parameters whose uncertainty has no remarkable effect on the model output uncertainty.

In this case, the failure rate associated to the degradation state 'Bad' turns out to be the parameter which the model is less sensitive to. Then, the uncertainty affecting this parameter is not considered and the nominal value (Table 2) is assigned to it.

Notice that the set of unavailability values reported in Columns 2 and 3 of Table 5 are not useful for representing the uncertainty on the unavailability, which must take into account not only the input parameter extreme values, but all the available information on the input parameter uncertainties, i.e., the possibility distributions. Thus, the sensitivity analysis here proposed cannot substitute the uncertainty representation and propagation tasks carried out in this work, but can be used to identify the input parameters whose uncertainty is most relevant. To further clarify this issue, we can say that the difference between the results in Table 5 and those of the approach proposed in this work to represent and propagate the uncertainty is twofold: on one hand, the PT-FRV based method is a global method to propagate the uncertainty [51]; on the other hand, the possibility distributions used to represent the uncertainty in the model parameters allow having a smaller commitment of the information provided by the expert.

Table 5: results of the local sensitivity analysis

\begin{tabular}{c|ccc|} 
Parameter & Minima & Maxima & $\Delta$ \\
\hline$\eta_{12}$ & $0.0142 \pm 1.2 \mathrm{e}-7$ & $0.0082 \pm 8.3 \mathrm{e}-8$ & $6.0 \mathrm{e}-3$ \\
$\beta_{12}$ & $0.0116 \pm 1.1 \mathrm{e}-7$ & $0.0108 \pm 8.8 \mathrm{e}-8$ & $0.8 \mathrm{e}-3$ \\
$\eta_{23}$ & $0.0119 \pm 1.0 \mathrm{e}-7$ & $0.0105 \pm 8.7 \mathrm{e}-8$ & $1.4 \mathrm{e}-3$ \\
$\beta_{23}$ & $0.0112 \pm 9.9 \mathrm{e}-8$ & $0.0110 \pm 1.2 \mathrm{e}-7$ & $0.2 \mathrm{e}-3$ \\
$\lambda_{1}$ & $0.0110 \pm 9.5 \mathrm{e}-8$ & $0.0114 \pm 9.6 \mathrm{e}-8$ & $0.4 \mathrm{e}-3$ \\
$\lambda_{2}$ & $0.0103 \pm 8.2 \mathrm{e}-8$ & $0.0144 \pm 1.4 \mathrm{e}-7$ & $4.1 \mathrm{e}-3$ \\
$\lambda_{3}$ & $0.0110 \pm 9.5 \mathrm{e}-8$ & $0.0111 \pm 8.8 \mathrm{e}-8$ & $0.1 \mathrm{e}-4$
\end{tabular}

Finally, notice that for every uncertain parameter and for any confidence level, the value considered in [46] is the middle point of the corresponding intervals provided by the expert.

\subsection{Possibilistic representation of the epistemic uncertainties}

Figure 11 reports the possibility distributions of the uncertain parameters of the case study, corresponding to the weighted families of Table 4. These are obtained by applying the procedure showed in Section 2.2.

\subsection{Uncertainty propagation}

Figure 12 shows the results obtained by applying the FRVs-based method to the considered case study. The Plausibility and Belief distributions of the portion of the mission time in which the component is in a down state are quite distant; this shows that for some favorable combinations of the uncertain parameters the system results to be much more available than for other combinations of the uncertain parameters which lead to high portions of downtime. Notice also that the Plausibility and Belief distributions bracket the CDF found the case in which the uncertainty on the model parameters is not accounted for (Section 3.3). 

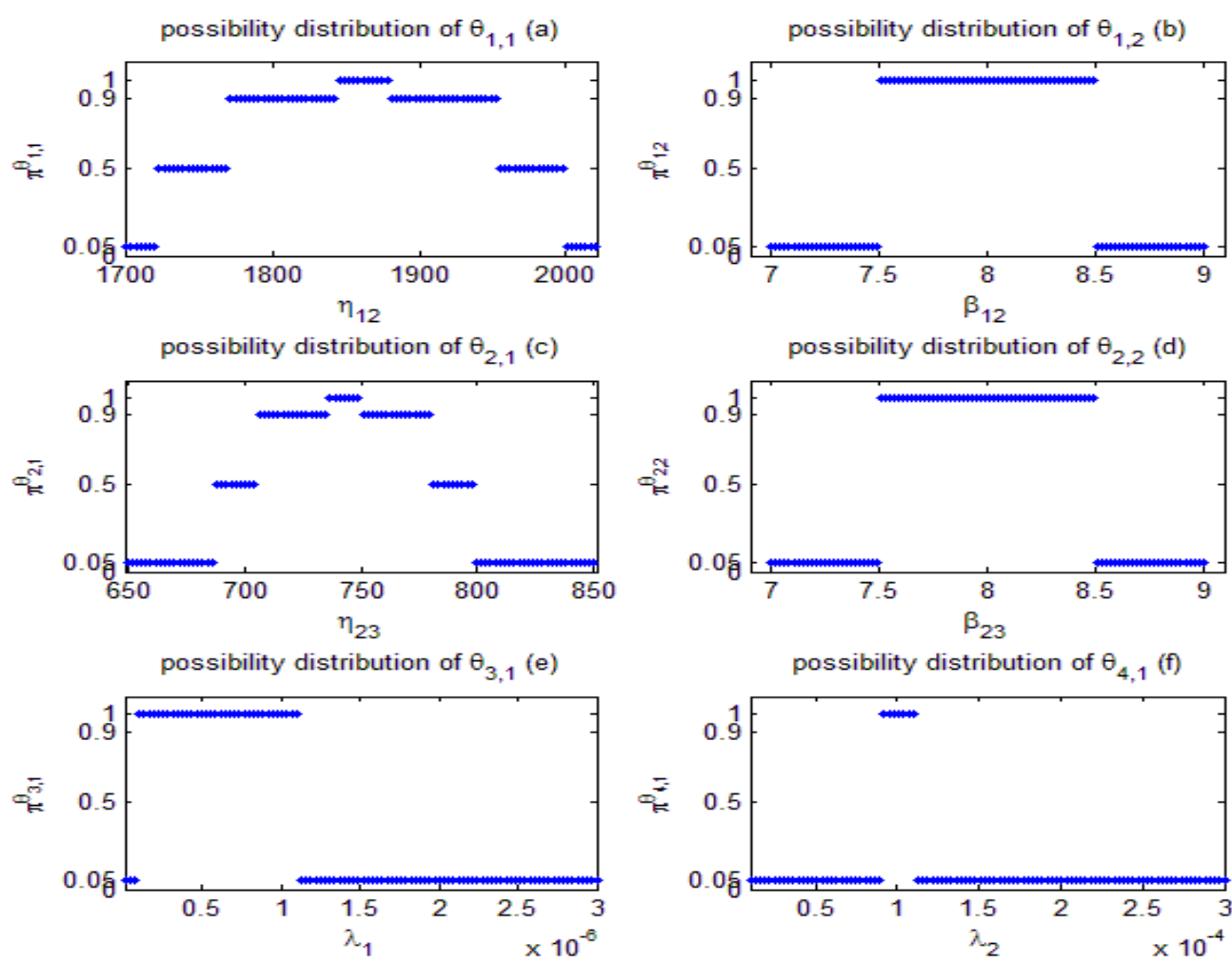

Figure 11: possibility distributions of the uncertain parameters of the case study

Notice that the results provided by the method discussed in this work are difficult to be interpreted. This is due to the fact that, differently from the case of no uncertainty, it is not possible to lump together the information provided by the method, i.e., the Plausibility and Belief function, into indicators such as their mean values which can be easily interpreted. This impossibility is due to the fact that the DSTE does not allow to define the mean value of an uncertain variable. However, in order to give an interpretation to the obtained results, one can focus on a given percentile of the belief and plausibility distributions. For example, the interval bounded by the values of the $95^{\text {th }}$ percentile of the Plausibility and Belief distributions is $[0.015$, 0.026]; the extremes of this interval constitute the lower and upper bounds, respectively, of the $95^{\text {th }}$ percentile of the portion of downtime in the mission time. In other words, this interval tells us that the $95 \%$ chance of the downtime of the component can be nor smaller than $1.5 \%$ nor larger than $2.6 \%$ of the mission time. Thus, if one is interested in the worst case, then he/she can assume that the $95^{\text {th }}$ percentile of the downtime is 0.026 , whereas in a more optimistic view, it can be valued at 0.015 . 


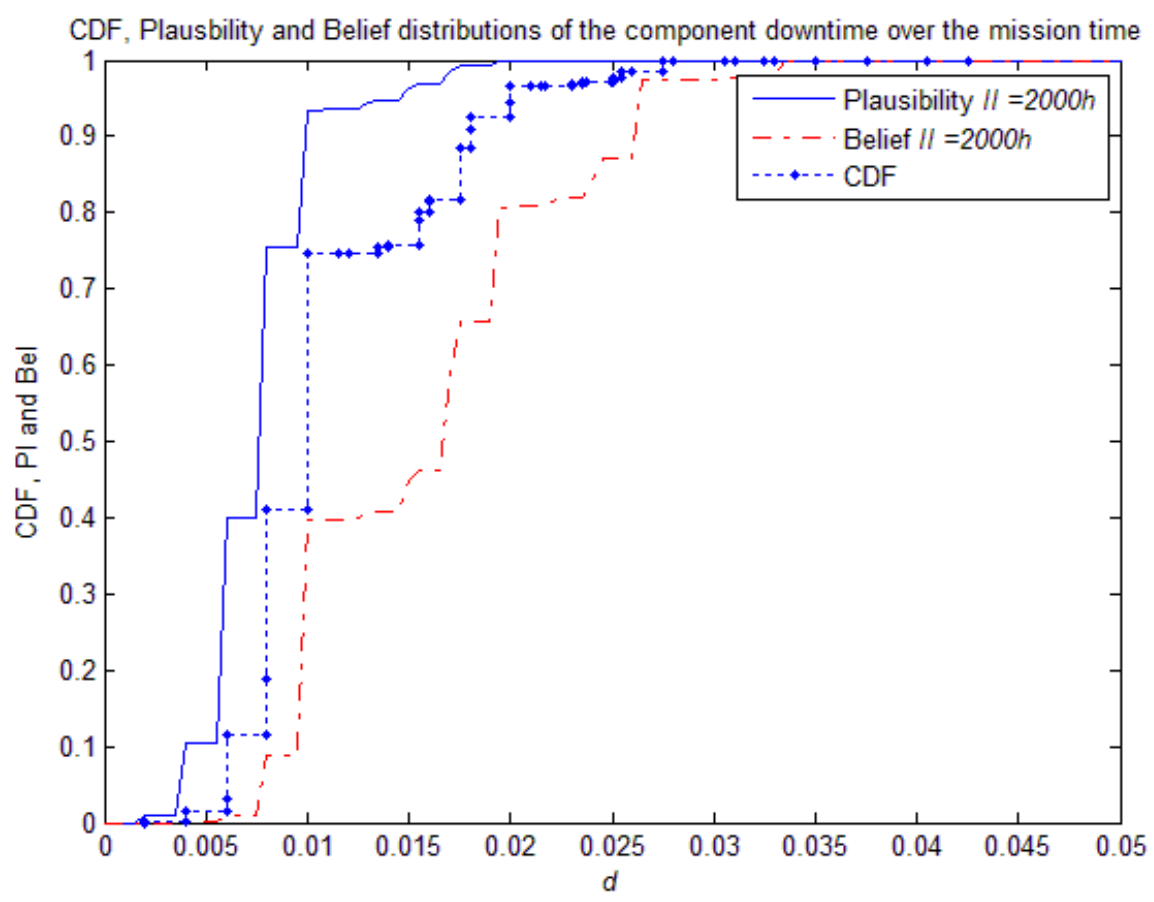

Figure 12: Plausibility and Belief distributions of the portion of mission time in which the component is unavailable, and the CDF corresponding to the case with no uncertainty.

Figure 13 and Figure 14 report the Plausibility and Belief distributions of the portion of downtime over the mission time and the total cost, respectively, corresponding to three different values of the $I I$, i.e., $I I=1000 \mathrm{~h}$, $I I=1500 \mathrm{~h}$ and $I I=2000 \mathrm{~h}$. These results do not lend themselves to an easy interpretation and do not allow to make a decision in a simple way. Indeed, while it is easy to state that inspecting the component every $1000 \mathrm{~h}$ is better than every $2000 \mathrm{~h}$, since these distributions are not overlapped, answering the question 'which value of the $I I$ is best?' is not trivial, as the distributions corresponding to $I I=1500 \mathrm{~h}$ and $I I=1000 \mathrm{~h}$ are overlapped. This calls for devising a method in support of maintenance decision makers, to help them get around these distributions. Notice also that the small amount of uncertainty on the values of both downtime and cost, when the component is inspected every $1000 \mathrm{~h}$, derives from the fact that the 'crowd' of the simulated MC component histories (i.e., the large number of components experiencing the same behavior) remains very compact in this case.

On the contrary, when the uncertainties affecting the parameters are not accounted for, the identification of the best value of $I I$ is more straightforward, since it is usually accepted to consider the mean value of the portion of mission time in which the component is faulty or the mean value of the cost as good indicators of the performance of the maintenance policy. 


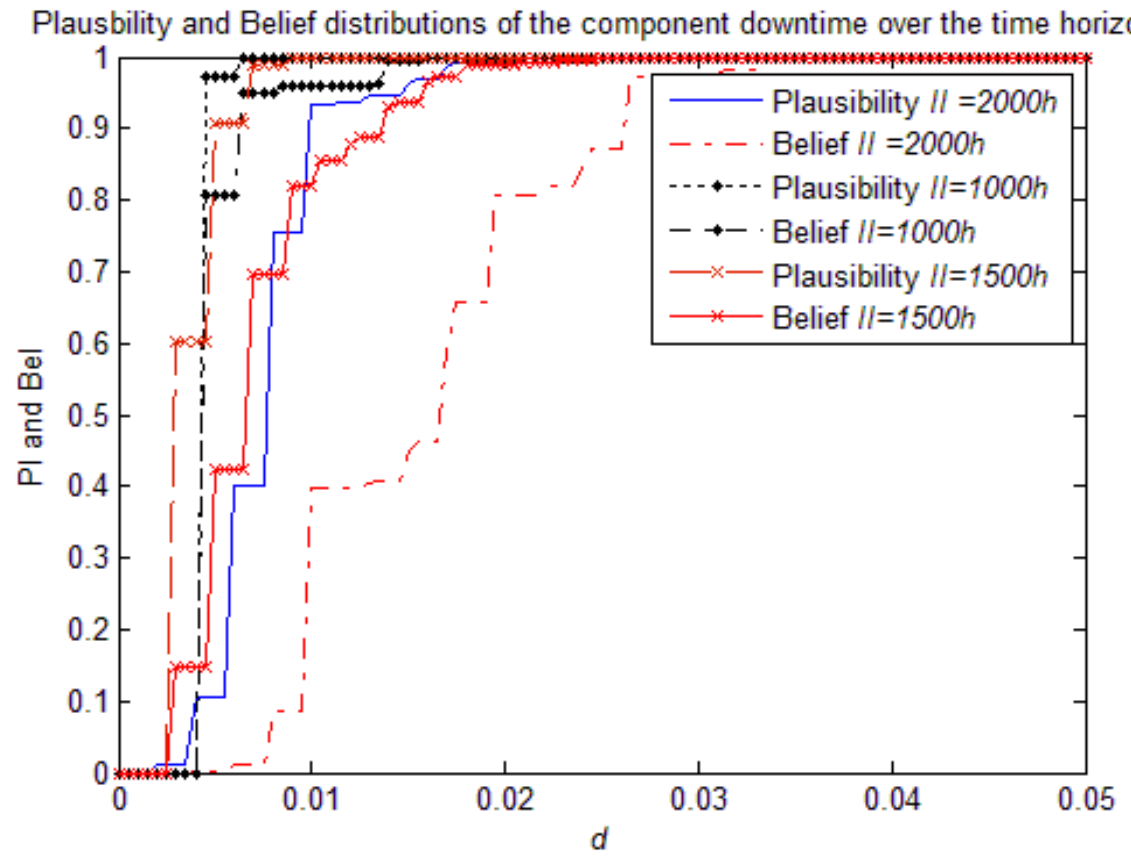

Figure 13: Plausibility and Belief distributions of the portion of time horizon in which the component is in a fault state, for different values of the control variable II

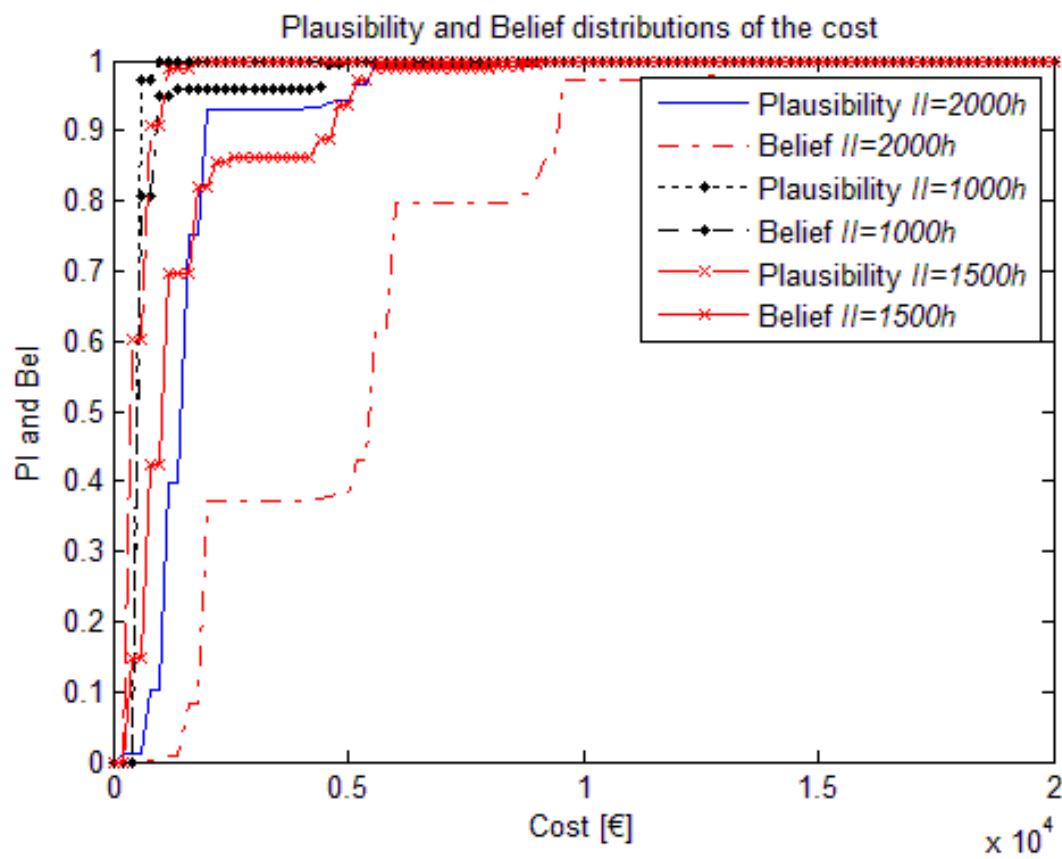

Figure 14: Plausibility and Belief distributions of the cost associated to the maintenance policy, for different values of the control variable II

\section{Conclusions}

Uncertainty affects the parameters of the models of the behavior of components subject to a given maintenance policy. An incorrect treatment of such uncertainty may lead to a serious bias in the outcomes of the analysis. In particular, such outcomes (e.g., occurrence probability of a failure scenario) may be biased in 
both conservative (e.g., estimated failure probability value larger than the actual one) and non-conservative (e.g., estimated failure probability value smaller than the actual one) directions. Often in practice the only information available on these parameters comes from experts, in an ambiguous and qualitative form. Most commonly, all that is known is that a certain value belongs to a certain interval [36]. The representation of the uncertainty of this information in terms of probability distributions would force a set of assumptions, with introduction of biases and loss of generality. In this work, a methodology has been proposed based on the following steps:

1) elicitation of the expert knowledge on the model parameters.

2) Representation of the uncertainty associated to the expert's judgment, avoiding introduction of unjustified, biasing assumptions. In this respect, notice that the choice of any probability distribution to represent the uncertainty in the expert's assignments would be absolutely arbitrary, if the expert is not able to assign such additional piece of information.

3) The propagation of the uncertainty on the maintenance performance indicators.

The methodology has been applied to a case study concerning the degradation model of a check valve of a turbo-pump lubricating system in a Nuclear Power Plant. The study has shown that neglecting uncertainty may drive the maintenance decision maker towards incorrect conclusions. In this case, if the unavailability computation were performed without taking into account the uncertainty on the input parameters, the decision maker would set the inspection intervals between maintenance actions to the value of $I I=1000 \mathrm{~h}$, whereas a proper consideration of the uncertainties through the use of FRV suggests that, on the basis of the available knowledge, this choice for the maintenance inspection interval is not better than other intervals such as $I I=1500 \mathrm{~h}$ and $I I=2000 \mathrm{~h}$.

The main current limitations of the methodology discussed in the present paper are:

- it is required that a single expert is knowledgeable, at least qualitatively, on all uncertain parameters and, what is more, is able to provide intervals of values for the uncertain parameters with associated confidence levels: this may be very difficult in practice. However, the FRVs-based method can be also applied when the expert provides just one interval of possible values per parameter, thus avoiding the problem of the confidence intervals.

- The results provided are difficult to be interpreted and managed. In this respect, a novel method has been proposed by the authors to compare the couples of Belief and Plausibility measures corresponding to two different solutions [52]. On this basis, an advanced extension of the Genetic Algorithms technique has been concocted to optimize maintenance problems in the presence of imprecision [28].

- Very large memory demand and computational times are required. Table 6 reports the computational times in case of 2000 samples and 8000 combinations of values of the uncertain parameters. However, being Matlab an interpretative language, a tool developed in other environments may be more performing. This issue will be tackled in future works.

- Further research effort needs to be spent in order to verify whether the procedure here illustrated can be interpreted as a conservative counterpart to the calculus of probabilistic variables under stochastic independence, due to the dependence between the choice of confidence levels.

\begin{tabular}{|l|l|}
\hline \multicolumn{1}{|c|}{ Parameters } & Values \\
\hline Number of FRVs & 2000 \\
\hline Number of combinations of uncertain parameters & 8000 \\
\hline CPU time (Intel Core 2 duo, $3.17 \mathrm{GHz}, 2 \mathrm{~GB}$ RAM) & $\approx 30 \mathrm{~h}$ \\
\hline
\end{tabular}

Table 6: FRVs-based method parameters 


\section{Acknowledgements}

This work has been performed thanks to the collaboration contract n. 5900051999 between Politecnico di Milano and Electricitè de France R\&D.

\section{References}

[1]. Guedes Soares, C., Caldeira Duarte, J., Garbatov, Y., Zio, E., Sorensen, J.D. 2010. Framework for maintenance planning. Safety and Reliability of Industrial Products, Systems and Structures. CRC Press/Balkema, Leiden, The Netherlands. C. Guedes Soares Ed.

[2]. Apostolakis, G.E. The concept of probability in safety assessments of technological systems. Science. Vol. 250, pp. 1359-1364. (1990)

[3]. Baudrit, C., Dubois, D., Perrot, N. Representing parametric models tainted with imprecision. Fuzzy Sets and Systems. Vol. 159, pp. 1913-1928. (2008)

[4]. Dubois, D. Possibility Theory and Statistical Reasoning. Computational Statistics and Data analysis. Vol. 51, pp. 47-69. (2006)

[5]. Groen, F.J., Mosleh, A. Foundations of probabilistic inference with uncertain evidence, International Journal of Approximate Reasoning. Vol. 39 (1), pp. 49-83. (2005)

[6]. Smets, P., Kennes, R. The Transferable Belief Model, Studies in Fuzziness and Soft Computing Vol. 219, pp 693-736. (2008)

[7]. Borgelt, C., Gebhardt, J., Kruse, R., Possibilistic Graphical Models. In: G. Della Riccia et al. Computational Intelligence in Data Mining, CISM Series, $N^{\circ} 408$, Springer, Berlin. (2000)

[8]. Bosc, P., Prade, H., An introduction to the fuzzy set and possibility theory-based treatment of soft queries and uncertain of imprecise databases. In: P. Smets, A. Motro, editors Uncertainty Management in Information Systems, Dordrecht: Kluwer, pp, 285-324. (1997)

[9]. Cayrac, D., Dubois, D., Haziza, M., Prade, H., Handling uncertainty with possibility theory and fuzzy sets in a satellite fault diagnosis application. IEEE Transactions on Fuzzy Systems, Vol. 4, pp. 251269. (1996)

[10]. Wolkenhauer, O. Possibility Theory with Applications to Data Analysis. Chichester: Research Studies Press. (1998)

[11]. Benferhat, T., Tabia, K., Classification with uncertain observations using possibilistic networks, In proceedings of 21st IEEE International Conference on Tools with Artificial Intelligence, pp. 493-499. (2009)

[12]. Clarke, N. D, McLeish, M.D., Vyn, T.J. Using certainty factors and possibilitytheory methods in a tillage selection expert system. Expert System with applications. Vol.4 (1), pp. 53-62. (1992)

[13]. Baraldi, P., Zio, E. A Combined Monte Carlo and Possibilistic Approach to Uncertainty Propagation in Event Tree Analysis. Risk Analysis, Vol. 28 (5), pp. 1309-1325, (2008)

[14]. Baraldi, P., Zio E. Event tree uncertainty analysis by Monte Carlo and possibility theory. in Martorell et al. (Eds), Safety, Reliability and Risk Analysis: Theory, Methods and Applications, Proceedings of the joint European Safety and Reliability (ESREL 2008) and Society for Risk Analysis Europe (17-th SRA-E) Conference, Valencia (Spagna), 22-25 September 2008, pp. 2101-2106. (2008)

[15]. Bloch, I. Some aspects of Dempster-Shafer evidence theory for classification of multi-modality medical images taking partial volume effect into account. Pattern Recognition Letters. Vol. 17 (8), pp. 905-919. (1996)

[16]. Srivastava, R.P., Mock, T., Belief Functions in Business Decisions, Physica-Verlag, Heidelberg. (2002) 
[17]. Parikh, C.R., Pont, M.J., Jones, N.B. Application of Dempster-Shafer theory in condition monitoring systems: A case study. Pattern Recognition Letters, Vol. 22 (6-7), pp. 777-785. (2001)

[18]. Schubert, J. Creating Prototypes for Fast Classification in Dempster-Shafer Clustering, in Qualitative and Quantitative Practical Reasoning, Procedings of the $1^{\text {st }}$ Int. Joint Conf. (ECSQARU-FAPR'97), Bad Honnef, Germany, 9-12 June 1997, pp. 525-535. (1997)

[19]. Hall, J.W., Soft Methods in Earth Systems Engineering, Advances in Soft Computing, Vol. 6, pp. 710. (2006)

[20]. Helton, J.C., Johnson, J.D., Oberkampf, W.L. An exploration of alternative approaches to the representation of uncertainty in model predictions. Reliability Engineering and System Safety, Vol. 85, pp. 39-71. (2004)

[21]. Baraldi, P., Compare, M., Zio, E. Maintenance policy performance assessment in presence of imprecision based on Dempster-Shafer Theory of Evidence, Information Sciences, Vol. 245, pp. 112131. (2013)

[22]. Deb, K., Gupta, S., Daum, D., Branke, J., Mall, A.K., Padmanabhan, D. Reliability-Based Optimization Using Evolutionary Algorithms. IEEE Transactions on Evolutionary Computation. Vol. 13 (5), pp. 1054-1074. (2009)

[23]. Eskandari, H., Geiger, C.D., Bird, R. Handling uncertainty in evolutionary multiobjective optimization: SPGA, in Proceedings of IEEE Congress on Evolutionary Computation, CEC 2007, 25-28 Sept. 2007, Singapore, pp. 4130 - 4137. (2007)

[24]. Hughes, E.J. Evolutionary multi-objective ranking with uncertainty and noise. Lecture Notes in Computer Science LNCS 1993, E. Zitzler et al. (Eds), Springer-Verlag Berlin Heidelberg. (2001)

[25]. Li, J., Kwan, R.S.K. A fuzzy genetic algorithm for driver scheduling. European Journal of Operational Research. Vol. 147 (2), pp. 334-344. (2003)

[26]. Trebi-Ollennu, A. White, B. A. Multiobjective Fuzzy Genetic Algorithm Optimization Approach to Nonlinear Control System Design. IEE Proceedings in Control Theory and Applications. Vol. 144 (2), pp. 137-142. (1997)

[27]. Limbourg, P. Multi-Objective Optimization of Problems with Epistemic Uncertainty. Lecture Notes in Computer Science LNCS, Vol. 3410, pp. 413-427. (2005)

[28]. Compare, M., Zio, E. Genetic algorithms in the framework of Dempster-Shafer Theory of Evidence for maintenance optimization problems, submitted to IEEE transactions on Reliability.

[29]. van Noortwijk, J.M. A survey of the application of gamma processes in maintenance. Reliability Engineering and System Safety. Vol. 94, pp. 2-21. (2009)

[30]. Wormsen, A. Härkegård, G. A statistical investigation of fatigue behaviour according to Weibull's weakest link theory. Proceedings of the 15th European Conference on Fracture, August 11-13, 2004, Stockholm, Sweden. (2004)

[31]. Paris, P.C., Erdogan, F. A critical analysis of crack propagation laws. Journal of Basic Engineering, Trans. ASME, 1963, Vol. 85, pp. 528-534. (1963)

[32]. Wang, H. A survey of maintenance policies of deteriorating systems. European Journal of Operational Research. Vol. 139, pp. 469-489. (2002)

[33]. Singpurwalla, N.D. Survival in Dynamic Environment. Statistical Science. Vol. 10, No. 1, pp. 86103. (1995)

[34]. Valdez-Flores, C., Feldman, R.M. A survey of preventive maintenance models for stochastically deteriorating single-unit systems. Naval Research Logistic. Vol. 36, pp. 419-446. (1989)

[35]. Wang, H., Pham, H. Some maintenance models and availability with imperfect maintenance models. Annals of Operations Research. Vol. 91, pp. 305-318. (1999)

[36]. Nicolai, R.P., Frenk, J.B.G., Dekker, R. Modeling and optimizing imperfect maintenance of coatings on steel structures. Structural Safety. Vol. 31, pp. 234-244. (2009)

[37]. Shapiro, A.F. Fuzzy Random Variables. Insurance Mathematics and Economics. Vol. 44 (2), pp. 307-314. (2009) 
[38]. Limbourg, P., de Racquigny, E. Uncertainty analysis using evidence theory- confronting level-1 and level-2 approaches with data availability and computational constraints. Reliability Engineering and System Safety. Vol. 95, pp. 550-564. (2010)

[39]. Mendel, J.M., John, R. I. B. Type-2 Fuzzy Stets Made simple. IEEE Transactions on Fuzzy Systems, Vol. 10 (2), pp. 117-127. (2002)

[40]. Baraldi, P., Compare, M., Zio, E. Dempster-Shafer theory of evidence to handle maintenance models tainted with imprecision. In proceedings of the $11^{\text {th }}$ International Probabilistic Safety Assessment and Management Conference and the Annual European Safety and Reliability Conference 2012, PSAM11 ESREL 2012. 25-29 June 2012, Helsinki, Finland (2012) 61-70.

[41]. Sandri, S.A., Dubois, D., Kalfsbeek, H.K. Elicitation, Assessement, and Pooling of Expert Judgements Using Possibility Theory. IEEE Transactions on Fuzzy Systems. Vol.3, pp.313-335. (1995)

[42]. Yang, L., Gao, Z., Li, K. Railway freight transportation planning with mixed uncertainty of randomness and fuzziness. Applied Soft Computing. Vol 11, No. 1. (2011)

[43]. Baudrit, C., Dubois, D., Guyonnet D. Joint Propagation and Exploitation of Probabilistic and Possibilistic Information in Risk Assessment. IEEE Transactions on Fuzzy Systems. Vol. 14, No. 5, pp. 593-608. (2006)

[44]. Teigen, K.H., Jorgensen, M. When $90 \%$ confidence intervals are only $50 \%$ certain: On credibility of credible intervals. Applied Cognitive Psychology. Vol. 19, pp. 455-475 (2005).

[45]. Zio E. "An Introduction to the Basics of Reliability and Risk Analysis", Singapore, World Scientific Publishing (2007)

[46]. Zille, V., Despujols, A., Baraldi, P., Rossetti, G., Zio, E. A framework for the Monte Carlo simulation of degradation and failure processes in the assessment of maintenance programs performance. Proceedings of the European Safety and Reliability Conference ESREL 2009, Praha, Czech Republic. (2009)

[47]. Remy, E., Idée, E., Briand, P., François, R. Bibliographical review and numerical comparison of statistical estimation methods for the three-parameters Weibull distribution. Proceedings of the European Safety and Reliability Conference ESREL 2010, Rhodes, Greece. (2010)

[48]. Marseguerra, M., Zio, E. Basics of Monte Carlo Method with application to System Reliability. LiLoLe-Verlag. (2002)

[49]. Baraldi, P., Zio, E. A comparison between probabilistic and Dempster-Shafer Theory approaches to Model Uncertainty Analysis in the Performance Assessment of Radioactive Waste Repositories. Risk Analysis. Vol. 30 (7), pp. 1139-1156. (2010)

[50]. Dubois, D., Prade, H. Possibility theory, probability theory and multiple valued-logics: A clarification. Annals o Mathematics in Artificial Intelligence. Vol. 32, pp. 35-66. (2001)

[51]. Zio, E. Computational Methods for Reliability and Risk Analysis, Singapore, World Scientific Publishing (2009).

[52]. Baraldi, P., Compare, M., Zio, E. Component ranking by birnbaum importance in presence of epistemic uncertainty in failure event probabilities. IEEE Transactions on Reliability. Vol. 62 (1), pp. $37-$ 48 (2013). 


\section{Appendix 1: Basics of DSTE and Possibility Theory}

\section{DSTE}

Belief functions can be used to process information which is at the same time of random and imprecise nature. The related formal DSTE (also called Theory of Belief Functions) involves the specification of a triplet $(S, I, m)$, where $S$ (called 'sample space') is the set that contains everything that could occur in the universe under consideration, $I$ (referred to as 'set of focal elements') is a countable collection of subsets of $S$, and $m$ (Basic Probability Assignment, BPA) is a function defined on subsets of $S$ such that:

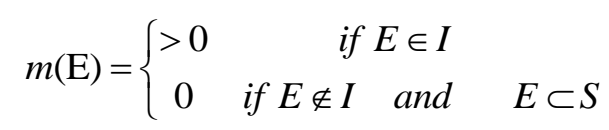

and

$\sum_{E \in I} m(E)=1$

More intuitively, the DSTE assigns weights (probability masses) to the focal sets; these weights represent the amount of likelihood that can be associated to the focal sets but to no proper subset of them (i.e., portions of these weights may move freely from one element of the focal set to another) [43].

The function $m$ is not the fundamental measure of likelihood of a proposition (set) A; rather, there are two measures of likelihood, called Belief and Plausibility, that are obtained from $m$ as [43]:

$$
\begin{aligned}
& \operatorname{Bel}(A)=\sum_{E \subseteq A} m(E) \\
& P l(A)=\sum_{E \cap A \neq 0} m(E)
\end{aligned}
$$

More intuitively, the belief in a proposition (set) $A$ is quantified as the sum of the probability masses assigned to all sets enclosed by it; hence, it is a lower bound representing the amount of belief that directly supports the proposition at least in part. The plausibility of event $A$ is, instead, the sum of the probability masses assigned to all sets whose intersection with the proposition is not empty; hence, it is an upper bound on the possibility that the proposition could be verified, i.e., it measures the fact that the proposition could possibly be true "up to that value" because there is only so much evidence that contradicts it [49].

\section{Possibility Theory}

In possibility theory, uncertainty is represented by means of a possibility function $\pi_{Y}(y)$, which expresses the degree of possibility of each value $y$ of the variable $Y$ in a set $S$. When $\pi_{Y}(y)=0$ for some $y$, it means that the outcome $y$ is considered an impossible situation. When $\pi_{Y}(y)=1$ for some $y$, it means that the outcome $y$ is possible, i.e., unsurprising, normal, usual [4]. These values are mutually exclusive, since the uncertain variable can take on only one true value. This also gives the normalization condition $\exists y \in S: \pi_{Y}(y)=1$, which is a claim that at least one value is viewed as totally possible, a much weaker statement than when probability is $1[50]$. 
A possibility distribution may also be viewed as a nested set of confidence intervals, which are the $\alpha$-cuts $[\underline{y}, \bar{y}]_{\alpha}=\left\{y \mid \pi_{Y}(y) \geq \alpha\right\}$ of $\pi$. The degree of certainty that $[\underline{y}, \bar{y}]_{\alpha}$ contains $Y$ is $N\left([\underline{y}, \bar{y}]_{\alpha}\right)\left(=1-\alpha\right.$ if $\pi_{Y}$ is continuous) [3].

The possibility and necessity measures $\Pi(A), N(A)$ for all subsets $A \subseteq S$ are defined by the associated possibility distribution $\pi_{Y}(y)$ through the following maximization and minimization relationships, respectively:

$\Pi(A)=\sup _{y \in A}\left\{\pi_{Y}(y)\right\}$

$N(A)=1-\Pi($ not $A)=1-\sup _{y \notin A}\left\{\pi_{Y}(y)\right\}$

Let $\mathbf{P}(\pi)$ be a family of probability distributions such that for all events $A, N(A) \leq P(A) \leq \Pi(A)$. Then,

$N(A)=\inf P(A)$ and $\Pi(A)=\sup P(A)$

where inf and sup are taken with respect to all probability measures in $\mathbf{P}$ [3]. Hence, the necessity measure is interpreted as a lower level for the probability and the possibility measure is interpreted as an upper limit. Referring to subjective probabilities, the bounds reflect that the analyst is not able or willing to precisely assign his/her probability, and the bounds are the best he/she can do given the information available; in other words, he or she can only describe a subset of $\mathbf{P}$ which contains his/her probability. 